\title{
A Chebyshev Spectral Method with Null Space Approach for Boundary-Value Problems of Euler-Bernoulli Beam
}

\author{
C. P. Hsu $\mathbb{D},{ }^{1,2}$ C. F. Hung $\mathbb{D}^{1},{ }^{1}$ and J. Y. Liao $\mathbb{D}^{2}$ \\ ${ }^{1}$ Department of Engineering Science and Ocean Engineering, National Taiwan University, No. 1, Sec. 4, Roosevelt Rd., Taipei, Taiwan \\ ${ }^{2}$ National Chung Shang Institute of Science and Technology, PB 90008-6-16, Longtang, Taoyuan, Taiwan \\ Correspondence should be addressed to C. F. Hung; hungef@ntu.edu.tw
}

Received 7 March 2018; Accepted 21 June 2018; Published 16 July 2018

Academic Editor: José J. Rangel-Magdaleno

Copyright (c) 2018 C. P. Hsu et al. This is an open access article distributed under the Creative Commons Attribution License, which permits unrestricted use, distribution, and reproduction in any medium, provided the original work is properly cited.

\begin{abstract}
We proposed a Chebyshev spectral method with a null space approach for investigating the boundary-value problem of a nonprismatic Euler-Bernoulli beam with generalized boundary or interface conditions. It is shown here that, with few vital improvements, a Chebyshev spectral collocation approach can be systematically applied to modeling nonprismatic Euler-Bernoulli beams with eigenvalue embedded tip-massed boundary conditions as well as the jump conditions that appear at the stepped interfaces. This study also presents a numerical stable asymptotic modal solution for the higher-order modes of a partially clamped beam and show that the proposed approach validates the robust higher-order modal solutions. Through a sequence of four increasingly complicated examples, using the proposed approach with higher-order modes, generalized boundary conditions, and interface jump conditions of nonprismatic beams, the results are in excellent agreement with those reported in the literature using various other approaches.
\end{abstract}

\section{Introduction}

Vibration analyses of Euler-Bernoulli (EB) beams are widely used in mechanical, civil, and aerospace engineering $[1,2]$. According to a variety of in situ applications, modal solutions of nonprismatic beams with generalized boundary conditions (BCs) are prerequisite in preliminary design. In addition to classical boundary conditions in many vibration textbooks, generalized boundary conditions such as damaged support, tip-mass, multiple-beams connected with elastic stiffness [3], and imperfect beams [4] have been adopted in many applications. For example, Olgac and Jalili [5] design a delayed resonator vibration absorber with a partially clamped beam. Moreover, beam models composed of a double layer carbon nanotube with elastically supported foundations and partially clamped boundary conditions are widely used to emulate the natural frequencies of integrated circuit assemblies under different external forces [6]. Recently, based on Jeong et al. [7], we developed a mechanically excited resonant beam for a pyroshock source simulator by using a partially clamped EB, and a configuration of a two-step beam is surveyed for the evaluation of the effects on adapter plates and test items.
Although there are a lot of papers in the literature that utilize nonclassical boundary conditions to analyze the free vibration of beams, they often tend to restrict their analysis to a low-order mode shapes, usually less than a dozen, while higher-order modes (more than 30) are rarely discussed. Nevertheless, Goncalves, Brennan and Elliott [8] investigate the numerical evaluation of higher-order modes of vibrations in uniform EB beams with various classical boundary conditions, and some asymptotic expressions are obtained. In this paper, a more general approach for partially clamped EB beams is presented and we obtain a unique set of asymptotic modal solution for higher-order modes. Cha, Wang, and Liao [9] analyzed chatter stability of surface grinder machines, modeled by a moving lumped grinding wheel element over an elastic worktable, with the last assumed to be a partially clamped EB beam. The assumed mode expansion is used for the EB beam, and asymptotic higher-order modes are required to facilitate the analysis.

The exact analytical solutions for free vibration of $\mathrm{EB}$ beams have been given only for prismatic beams with simply supported boundary conditions. Lai, Hsu, and Chen $[10,11]$ use a modified Adomian decomposition method (ADM) 
to analyze the modal solutions of a tip-massed tapered beam. However, the tip-masses at the ends of the beam introduce eigenvalue embedded boundary conditions that further complicate the modal solutions of the tip-massed EB beam. In addition, Mao and Pietrzko [12] discuss the modal solutions of a stepped beam using ADM. For a beam with a discontinuous cross-section, together with the boundary conditions at both ends, there are also interface conditions at the step location of the two beam segments. However, the interface conditions must obey displacement compatibility and force equilibrium relationships. Again, Mao discusses the modal solutions of nonprismatic EB beams for piezoelectric modal sensors by using the transformation matrix method of [13]. Moreover, the modal solutions of a stepped beam with multiple cracks and different boundary conditions are discussed in Attar [14]. Lastly, Sarkar and Ganguli [15] provide a modal tailoring and closed-form solutions for a rotating nonprismatic beam by using an inverse problem approach.

Besides the above-mentioned approach, the spectral method is another numerical approach for EB beams, which can potentially provide superior accuracy and domain flexibility $[16,17]$. Because of the superior convergence of the Chebyshev spectral method (CSM), it has been widely used for the modal solution of various Timoshenko beams [1821]. However, in the Mathematical Institute at University of Oxford, Professor Trefethen has initiated and held the Chebfun project since 2002. The Chebfun toolbox under the MATLAB environment enables us to formulate the spectral collocation matrix automatically for the integral and integrodifferential equations [22-27]. Driscoll, Bornemann, and Trefethen [25] solve linear differential equations based on the developed Chebfun toolbox with the object-oriented MATLAB symbolic scripts and introduce the "chebop" system, where the ODE and boundary conditions may be represented with symbolic chebop commands.

For boundary and interface conditions issues that arise in CSM-type approaches, Driscoll and Hale [26] suggest a rectangular spectral collocation method (RSC) without any rows deletions to formulate the corresponding CSM. Moreover, Smith, Laoulache, and Heryudono [28] focus on the boundary conditions of CSM and discuss the row replacement method, the fictitious point method, and the rectangular collocation method, all implemented as velocity and vorticity conditions for solving the unsteady NavierStokes equations of an annular Couette flow.

As in the classical boundary conditions and low-order modal solutions, the eigensolutions of CSM may be easily obtained by using the overloaded "eigs" command in the Chebfun toolbox. However, in some cases such as with nonclassical boundary conditions and higher-order modes, it is not always working well. These nonclassical boundary conditions may result in an ill-conditioned matrix and numerical instability in the eigensolutions. Wilkinson [29] explains the reasons of an ill-condition matrix and Saad [30] provides a typical preconditioned iterative approach for computing large eigenvalue problems. Since in the null space approach we project the original solution space to its constrained space instead, many null space applications such as solving nonlinear system of equations [31], data reconciliation, and variable classification have been applied in of Mitsas [32]. Considering that the rigid-body modes are in the null space of the matrix of the structure system, Felippa and Park [33] apply the null space approach to the free-free flexibility matrix of the structures and explore the relationship between the rigidbody modes and boundary conditions.

The main purpose of this paper is to propose a systematic procedure using the Chebyshev spectral method with the null space approach (NSA) for the ill-conditioned system matrix caused by modeling the nonprismatic EB beams with generalized boundary and interface conditions. Another contribution of this paper is to introduce the chebop system in the Chebfun toolbox for modeling the governing equation with boundary or interface conditions of EB beams. The symbolic operator commands in the chebop are dramatically simple and one may represent the governing equation and boundary or interface conditions of an EB beam in just a few lines long executable MATLAB code. After constructing the CSM collocated matrix, one may use the NSA to project the ill-conditioned matrix to the null space of the boundary or interface conditions, thereby significantly improving the condition of the spectral collocation matrix. For validation purposes, the exact modal solutions of the simply supported EB beam are used to benchmark the accuracy of higher-order modal solutions by CSM with NSA. This paper also derives higher-order asymptotic modal solutions for the partially clamped EB beam and benchmarks the accuracy of the derived asymptotic solution with the proposed approach. Finally, several examples of a nonprismatic EB beam with generalized boundary and interface conditions, such as tipmassed and stepped beams, are used to demonstrate the proposed CSM with NSA approach. Those examples show the straightforward symbolic formulation in accordance with the governing equation and boundary conditions of an EB beam, in obtaining the robust eigensolutions of boundaryvalue problems.

\section{Governing Equation and Boundary Conditions of EB Beam}

2.1. The Governing Equation of a Nonprismatic EB Beam with Tip-Masses of Rotary Inertia and Nonclassical Boundary Conditions. Consider a nonprismatic EB beam as shown in Figure 1, whose length is $l$ with both ends restrained by the translation and torsion springs. At the both ends of the beam, there are concentrated masses that comprise the rotatory inertia of the mass.

The equation of motion for the transverse vibration of the beam is given by

$$
\frac{\partial^{2}}{\partial x^{2}}\left[E I(x) \frac{\partial^{2} y(x, t)}{\partial x^{2}}\right]+\rho A(x) \frac{\partial^{2} y(x, t)}{\partial t^{2}}=0,
$$

where $y(x, t)$ denotes the transverse deflection of the beam, $A(\mathrm{x})$ is the cross-sectional area at $x, I(x)$ is the area moment of inertia of $A(x)$, and $\rho$ and $E$ are the mass density (mass per unit volume) and Young's modulus, respectively. 


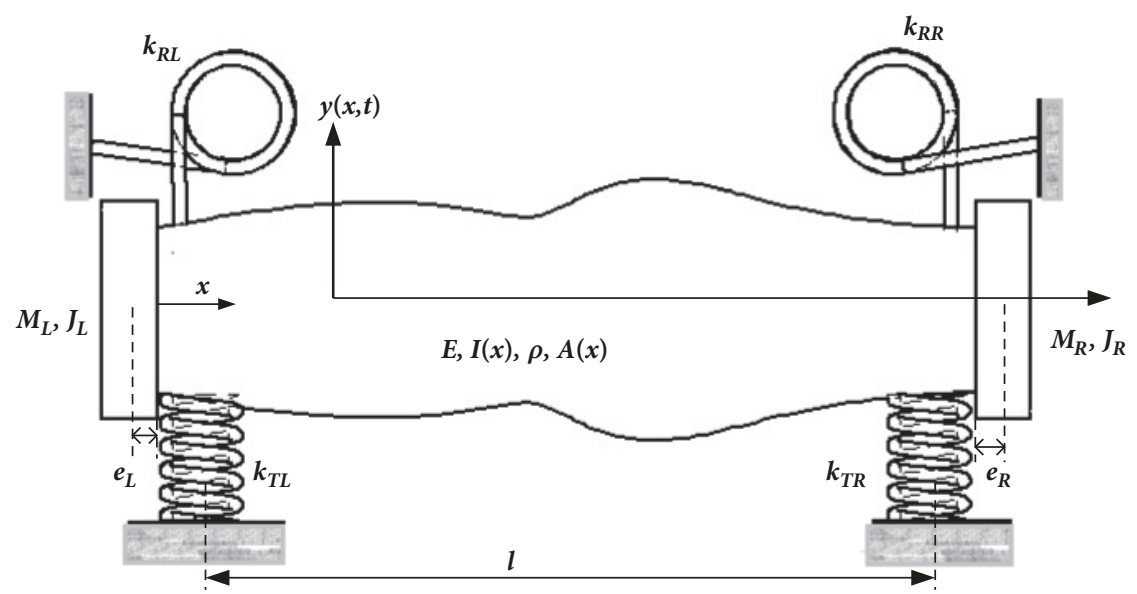

FIGURE 1: A nonprismatic EB beam with elastically restrained ends, which supports tip-masses of rotatory inertia at both ends.

Assuming the beam is oscillating harmonically at a frequency $\omega(\mathrm{rad} / \mathrm{s})$ and the solutions of transverse deflection to (1) are

$$
y(x, t)=\phi(x) e^{-i \omega t},
$$

(1) becomes

$$
\frac{d^{2}}{d x^{2}}\left[E I(x) \frac{d^{2} \phi(x)}{d x^{2}}\right]-\omega^{2} \rho A(x) \phi(x)=0 .
$$

The corresponding external boundary conditions at both ends are given by

$$
\begin{aligned}
& E I(x) \frac{d^{2} \phi(x)}{d x^{2}}+\left(J_{L}+M_{L} e_{L}^{2}\right) \omega^{2} \frac{d \phi(x)}{d x}-k_{R L} \frac{d \phi(x)}{d x} \\
& \quad-M_{L} e_{L} \omega^{2} \phi(x)=0, \\
& \frac{d}{d x}\left[E I \frac{d^{2} \phi(x)}{d x^{2}}\right]+M_{L} e_{L} \omega^{2} \frac{d \phi(x)}{d x}+k_{T L} \phi(x) \\
& \quad-M_{L} \omega^{2} \phi(x)=0,
\end{aligned}
$$

at $x=0$, and

$$
\begin{aligned}
& E I \frac{d^{2} \phi(x)}{d x^{2}}+k_{R R} \frac{d \phi(x)}{d x}-\left(J_{R}+M_{R} e_{R}^{2}\right) \omega^{2} \frac{d \phi(x)}{d x} \\
& \quad-M_{R} e_{R} \omega^{2} \phi(x)=0, \\
& \frac{d}{d x}\left[E I \frac{d^{2} \phi(x)}{d x^{2}}\right]+M_{R} e_{R} \omega^{2} \frac{d \phi(x)}{d x}-k_{T R} \phi(x) \\
& \quad+M_{R} \omega^{2} \phi(x)=0,
\end{aligned}
$$

at $x=l$.

Here, $k_{T R}, k_{R R}, M_{R}, J_{R}, e_{R}$ and $k_{T L}, k_{R L}, M_{L}, J_{L}, e_{L}$, borrowed from the nomenclatures of [9], are the translational springs, the torsion springs, the concentrated masses attached at beam tip, the moments of inertia of the tip-masses, and the eccentricities which are the distances between the beam tip and the center of the tip-mass at the right end and left end of the beam, respectively.

Equations (3) to (7) can be converted to a nondimensional form by introducing the following quantities:

$$
\begin{aligned}
& X=\frac{x}{l} \text {; } \\
& \Phi(X)=\frac{\phi(x)}{l} ; \\
& \Omega^{4}=\frac{\rho A_{0} \omega^{2} l^{4}}{E I_{0}} \\
& \mu_{m L}=\frac{M_{L}}{\rho A_{0} l} ; \\
& \mu_{m R}=\frac{M_{R}}{\rho A_{0} l} ; \\
& K_{R L}=\frac{k_{R L} l}{E I_{0}} ; \\
& K_{T L}=\frac{k_{T L} l^{3}}{E I_{0}} ; \\
& K_{R R}=\frac{k_{R R} l}{E I_{l}} ; \\
& K_{T R}=\frac{k_{T R} l^{3}}{E I_{l}} ; \\
& \gamma_{L}=\sqrt{\frac{J_{L}}{M_{L} l^{2}}} ; \\
& \gamma_{R}=\sqrt{\frac{J_{R}}{M_{R} l^{2}}} ; \\
& \delta_{L}=\frac{e_{L}}{l} ;
\end{aligned}
$$




$$
\begin{gathered}
\delta_{R}=\frac{e_{R}}{l} ; \\
i(X)=\frac{I(x)}{I_{0}} ; \\
a(X)=\frac{A(x)}{A_{0}},
\end{gathered}
$$

where the range of $X$ is $[0,1]$, and $A_{0}$ and $I_{0}$ are the crosssectional area and the moment of inertia at $x=0$, respectively. is

Substituting (8) into (3), the nondimensional form of (3)

$$
\frac{d^{2}}{d X^{2}}\left[i(X) \frac{d^{2} \Phi(X)}{d X^{2}}\right]-\Omega^{4} a(X) \Phi(X)=0
$$

Using (8) in (4) to (7), the boundary conditions at both ends in nondimensional form become as follows:

$$
\begin{aligned}
& \Phi^{\prime \prime}(0)+\left[\mu_{m L}\left(\delta_{L}^{2}+\gamma_{L}^{2}\right) \Omega^{4}-K_{R L}\right] \Phi^{\prime}(0) \\
& -\mu_{m L} \delta_{L} \Omega^{4} \Phi(0)=0, \\
& \Phi^{\prime \prime \prime}(0)-i^{\prime}(0) \Phi^{\prime \prime}(0)+\mu_{m L} \delta_{L} \Omega^{4} \Phi^{\prime}(0) \\
& +\left(K_{T L}-\mu_{m L} \Omega^{4}\right) \Phi(0)=0, \\
& \Phi^{\prime \prime}(1)+\left[K_{R R}-\mu_{m R}\left(\delta_{R}^{2}+\gamma_{R}^{2}\right) \Omega^{4}\right] \Phi^{\prime}(1) \\
& -\mu_{m R} \delta_{R} \Omega^{4} \Phi(1)=0, \\
& \Phi^{\prime \prime \prime}(1)-\frac{i^{\prime}(1)}{i(1) \Phi^{\prime \prime}(1)}+\mu_{m R} \delta_{R} \Omega^{4} \Phi^{\prime}(1) \\
& -\left(K_{T R}-\mu_{m R} \Omega^{4}\right) \Phi(1)=0,
\end{aligned}
$$

where

$$
\begin{aligned}
\Phi^{\prime}(X) & =\frac{d \Phi(X)}{d X}, \\
\Phi^{\prime \prime}(X) & =\frac{d^{2} \Phi(X)}{d X^{2}}, \\
\Phi^{\prime \prime \prime}(X) & =\frac{d^{3} \Phi(X)}{d X^{3}}, \\
i^{\prime}(X) & =\frac{d i(X)}{d X} .
\end{aligned}
$$

2.2. The Governing Equation of a Stepped EB Beam with Nonclassical Boundary Conditions. Consider the free vibration of a straight EB beam consisting of $N$ uniform sections elastically restrained at both ends, as shown in Figure 2. The total length is $l$, and the ratio of the distance from the right end of the $S_{j}$ section to the left end of the beam is $R_{i}$ $(i=1,2, \ldots, N)$. Let us define the junction parameters $\eta_{j}, \gamma_{j}$, and $\mu_{j}$ for the beam sections $j$ and $j+1(j=1,2, \ldots, N-1)$ as follows:

$$
\begin{aligned}
& \eta_{j}=\frac{E_{j+1} I_{j+1}}{E_{j} I_{j}}, \\
& \gamma_{i}=\frac{\rho_{j+1} A_{j+1}}{\rho_{j} A_{j}}, \\
& \mu_{j}=\frac{\gamma_{j}}{\eta_{j}},
\end{aligned}
$$

for $j=1,2, \cdots, N-1$.

Here $E_{j}, \rho_{j}, A_{j}$, and $I_{j}$ are Young's modulus, density per length, the cross-section area, and the inertia of cross-section area of $j$-th section, respectively. Assuming the stepped beam is oscillating harmonically at a frequency $\omega(\mathrm{rad} / \mathrm{s})$, the nondimensional governing equation for each segment can be written as

$$
\frac{d^{4} \Phi_{i}\left(X_{i}\right)}{d X_{i}^{4}}-\Omega_{i}^{4} \Phi_{i}\left(X_{i}\right)=0,
$$

$$
X_{i} \in\left[0, R_{i}\right], i=1,2, \cdots, N \text {, }
$$

where $\Omega_{i}^{2}=\omega \sqrt{\rho_{i} A_{i} l^{4} / E_{i} I_{i}}$, and $\Omega_{j}^{2}=\mu_{j} \Omega_{j+1}^{2}, j=1, \ldots, N-$ 1.

The interface conditions at the junction of each segment are

$$
\begin{aligned}
\text { identical jump: } \Phi_{i}\left(R_{j}\right) & =\Phi_{i+1}\left(R_{j}\right), \\
\Phi_{i}^{\prime}\left(R_{j}\right) & =\Phi_{i+1}^{\prime}\left(R_{j}\right), \\
\text { scaled jump: } \Phi_{i}^{\prime \prime}\left(R_{j}\right) & =\eta_{j} \Phi_{i+1}^{\prime \prime}\left(R_{j}\right), \\
\Phi_{i}^{\prime \prime \prime}\left(R_{j}\right) & =\eta_{j} \Phi_{i+1}^{\prime \prime \prime}\left(R_{j}\right),
\end{aligned}
$$

and the boundary conditions at the both ends are

$$
\begin{array}{r}
\Phi_{1}^{\prime \prime}(0)=K_{R L} \Phi_{1}^{\prime}(0), \\
\Phi_{1}^{\prime \prime \prime}(0)=-K_{T L} \Phi_{1}(0), \\
\eta_{N-1} \Phi_{N}^{\prime \prime}(1)=-K_{R R} \Phi_{N}^{\prime}(1), \\
\eta_{N-1} \Phi_{N}^{\prime \prime \prime}(1)=K_{T R} \Phi_{N}(1) .
\end{array}
$$

2.3. Numerical Instability in Higher-Order Modes of an EB Beam for Partially Clamped Boundary Conditions. This section derives an asymptotic solution for the higher-order modes of a partially clamped EB beam by using trigonometric and modified hyperbolic functions. The evaluation of hyperbolic functions [8] in the analytical solutions of higher-order modes of a partially clamped EB beam will cause numerical instability issues. The numerical ill-conditions occur in the argument of the hyperbolic functions and lead to a quantity larger than that can be represented by the machine. We derive the analytical solution of a partially clamped EB beam 


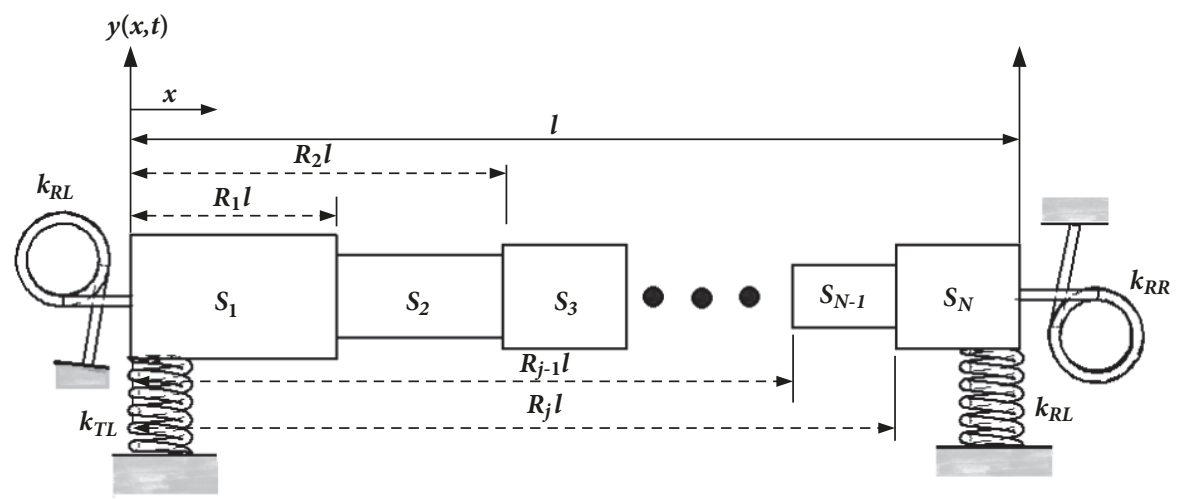

Figure 2: A stepped beam with elastically restrained ends.

and its asymptotic forms for higher-order modes. A detailed derivation is given in the Appendix for the interested reader.

The governing equation of a partially clamped uniform EB beam is shown in (1). The partially clamped condition may be represented by elastically restrained translation and torsion springs. The corresponding boundary conditions at both ends of the beam can be expressed as

$$
\begin{gathered}
M_{x=0}=E I\left(\phi^{\prime \prime}\right)_{x=0}=k_{\theta L}\left(\phi^{\prime}\right)_{x=0}, \\
V_{x=0}=E I\left(\phi^{\prime \prime \prime}\right)_{x=0}=-k_{T L}(\phi)_{x=0}, \\
M_{x=l}=E I\left(\phi^{\prime \prime}\right)_{x=l}=-k_{\theta R}\left(\phi^{\prime}\right)_{x=l}, \\
V_{x=l}=E I\left(\phi^{\prime \prime \prime}\right)_{x=l}=k_{T R}(\phi)_{x=l} .
\end{gathered}
$$

Specifically, $\phi$ is written as a finite sum, the so-called Galerkin approximation:

$$
\phi(x)=\sum_{i=1}^{n} q_{i} \psi_{i}(x) .
$$

The assumed mode expansion of $\psi_{i}(x)$ can be written in terms of trigonometric and hyperbolic functions as

$$
\begin{aligned}
\psi_{i}(x)= & C_{1} \sin \left(\kappa_{i} x\right)+C_{2} \cos \left(\kappa_{i} x\right)+C_{3} \sinh \left(\kappa_{i} x\right) \\
& +C_{4} \cosh \left(\kappa_{i} x\right), \\
\kappa_{i}^{2}= & \omega_{i} \sqrt{\frac{\rho A}{E I}} .
\end{aligned}
$$

Let us define the following nondimensional spring constants,

$$
\begin{aligned}
& \beta_{1}=\frac{k_{\theta L} l}{E I}, \\
& \beta_{2}=\frac{k_{T L} l^{3}}{E I}, \\
& \beta_{3}=\frac{k_{\theta R} l}{E I}, \\
& \beta_{4}=\frac{k_{T R} l^{3}}{E I} .
\end{aligned}
$$

The boundary conditions in (19) can be given by the following nondimensional forms.

$$
\begin{aligned}
\psi_{i}^{\prime \prime}(0)-\frac{\beta_{1}}{l} \psi_{i}^{\prime}(0) & =0, \\
\psi_{i}^{\prime \prime \prime}(0)+\frac{\beta_{2}}{l^{3}} \psi_{i}(0) & =0, \\
\psi_{i}^{\prime \prime}(l)+\frac{\beta_{3}}{l} \psi_{i}^{\prime}(l) & =0, \\
\psi_{i}^{\prime \prime \prime}(l)-\frac{\beta_{4}}{l^{3}} \psi_{i}(l) & =0 .
\end{aligned}
$$

According to descriptions found in the Appendix, the analytical modal solution of the beam with partially clamped boundary conditions can be described by

$$
\begin{aligned}
\psi_{i, \text { exact }}(x)=\sin \left(k_{i} x\right)\left[\left(C-C_{H}\right) \Lambda_{i}^{5}+\left(S-S_{H}\right) \beta_{3} \Lambda_{i}^{4}\right. \\
+2 \beta_{2} S_{H} \Lambda_{i}^{2}+\left(\beta_{1} C_{H}+\beta_{1} C+2 \beta_{3} C_{H}\right) \beta_{2} \Lambda_{i} \\
\left.+\left(S_{H}+S\right) \beta_{1} \beta_{2} \beta_{3}\right]+\cos \left(k_{i} x\right)\left[\left(S_{H}-S\right) \Lambda_{i}^{5}\right. \\
+\left(2 \beta_{1} C_{H}+\beta_{3} C_{H}+\beta_{3} C\right) \Lambda_{i}^{4}+2 \beta_{2} \beta_{3} S_{H} \Lambda_{i}^{3} \\
\left.+\left(S_{h}+S\right) \beta_{1} \beta_{2} \Lambda_{i}-\left(C_{H}+C\right) \beta_{1} \beta_{2} \beta_{3}\right] \\
+\sinh \left(k_{i} x\right)\left[\left(C-C_{H}\right) \Lambda_{i}^{5}+\left(S-S_{H}\right) \beta_{3} \Lambda_{i}^{4}\right. \\
+2 \beta_{2} S \Lambda_{i}^{2}-\left(\beta_{1} C+\beta_{1} C_{H}+2 \beta_{3} C\right) \beta_{2} \Lambda_{i} \\
\left.+\left(S+S_{H}\right) \beta_{1} \beta_{2} \beta_{3}\right]+\cosh \left(k_{i} x\right)\left[\left(S_{H}-S\right) \Lambda_{i}^{5}\right. \\
+\left(2 \beta_{1} C+\beta_{3} C_{H}+\beta_{3} C\right) \Lambda_{i}^{4}+2 \beta_{1} \beta_{3} S \Lambda_{i}^{3} \\
\left.+\left(S_{H}+S\right) \beta_{1} \beta_{2} \Lambda_{i}+\left(C_{H}-C\right) \beta_{1} \beta_{2} \beta_{3}\right]
\end{aligned}
$$

where $\Lambda_{i}=k\left(\omega_{i}\right) l=k_{i} l$ is a nondimensional natural frequency, $S, C, S_{H}$, and $C_{H}$ represent $\sin \left(\Lambda_{i}\right), \cos \left(\Lambda_{i}\right), \sinh \left(\Lambda_{i}\right)$, and $\cosh \left(\Lambda_{i}\right)$, respectively.

However, (24) is numerically unstable using double precision floating point arithmetic with MATLAB for mode order greater than 13, due to the presence of hyperbolic 
functions (e.g., MATLAB cannot tell the difference between $\sinh 13 \pi-1$ and $\sinh 13 \pi)$. The key step in approximating (24) for higher-order mode shapes is to use the modified exponent functions replacing the original hyperbolic functions. This step can solve the problem of divergence of the analytical solution while preserving the orthogonality of the higherorder modes. Refer to the Appendix for the detailed higherorder asymptotic modal solutions. The asymptotic solution for higher-order mode shapes of a uniform EB beam with partially clamped at both ends is shown below:

$$
\begin{aligned}
& \psi_{i, \text { asymp }}(x)=\sin \left(k_{i} x\right)\left[-\Lambda_{i}^{5}-\Lambda_{i}^{4} \beta_{3}+2 \beta_{2} \Lambda_{i}^{2}\right. \\
& \left.+\left(\beta_{1} \beta_{2}+2 \beta_{3} \beta_{2}\right) \Lambda_{i}+\beta_{3} \beta_{2} \beta_{1}\right]+\cos \left(k_{i} x\right)\left[\Lambda_{i}^{5}\right. \\
& \left.+\left(\beta_{3}+2 \beta_{1}\right) \Lambda_{i}^{4}+2 \beta_{1} \beta_{3} \Lambda_{i}^{3}-\beta_{1} \beta_{2} \Lambda_{i}-\beta_{3} \beta_{2} \beta_{1}\right] \\
& +\mathrm{e}^{-k_{i} x}\left(\Lambda_{i}^{5}+\Lambda_{i}^{4} \beta_{3}+\beta_{1} \beta_{2} \Lambda_{i}+\beta_{3} \beta_{2} \beta_{1}\right) \\
& +\mathrm{e}^{-k_{i}(l-x)}\left\{(C-S) \Lambda_{i}^{5}+\left[\beta_{3}(S+C)+2 \beta_{1} C\right] \Lambda_{i}^{4}\right. \\
& +2 \beta_{1} \beta_{3} S \Lambda_{i}^{3}+\beta_{2} S \Lambda_{i}^{2}+\beta_{2}\left[\beta_{1}(S-C)-2 \beta_{3} C\right] \Lambda_{i} \\
& \left.\quad-\beta_{1} \beta_{2} \beta_{3}(S+C)\right\} .
\end{aligned}
$$

To tackle the numerical error issue indicated in [8], we apply (24) with $\Lambda_{i} \leq 6 \pi$ instead of $\Lambda_{i} \leq 13 \pi$ as a breakpoint to compute the lower-order analytical solutions and the higherorder asymptotic solutions. Therefore, for $\Lambda_{i} \leq 6 \pi$, use (24) to obtain the mode shapes; otherwise use (25).

\section{The Chebyshev Spectral Method and the Differential Operator in ODE}

From [16], the $k$ th-order Chebyshev polynomial $T_{N}(s)$ and Chebyshev-Gauss-Lobatto (CGL) collocation points $s_{j}$, in the domain of $s_{j} \in[-1,1]$, can be expressed, respectively, by

$$
T_{N}\left(s_{j}\right)=\cos \left[N\left(\cos ^{-1} s_{j}\right)\right], \quad s_{j}=\cos \left(\frac{j \pi}{N}\right) .
$$

The derivative of a smooth $g(x)$ function at the CGL points $s_{j}$ can then be computed via matrix-vector multiplication, which can be formally represented as

$$
\left\{\begin{array}{c}
g^{\prime}\left(s_{0}\right) \\
g^{\prime}\left(s_{1}\right) \\
\vdots \\
g^{\prime}\left(s_{N}\right)
\end{array}\right\}=\left[D_{N}\right]\left\{\begin{array}{c}
g\left(s_{0}\right) \\
g\left(s_{1}\right) \\
\vdots \\
g\left(s_{N}\right)
\end{array}\right\},
$$

where $\left[D_{N}\right]$ is a $(N+1) \times(N+1)$ matrix.

The elements within the matrix can be expressed as

$$
\begin{aligned}
& \left(D_{N}\right)_{00}=\frac{\left(2 N^{2}+1\right)}{6}, \\
& \left(D_{N}\right)_{N N}=-\left(D_{N}\right)_{00},
\end{aligned}
$$

$$
\begin{aligned}
& \left(D_{N}\right)_{j j}=\frac{-s_{j}}{\left[2 \sin ^{2}(j \pi / N)\right]}, \quad j=1, \ldots, N-1, \\
& \left(D_{N}\right)_{i j} \\
& =\frac{(-1)^{i+j+1} c_{i}}{\left[2 c_{j} \sin (2 \pi / N)(i+j) \sin (2 \pi / N)(i-j)\right]}, \\
& \quad i \neq j, i, j=0, \ldots, N-1,
\end{aligned}
$$$$
c_{j}= \begin{cases}2, & j=0 \text { or } N \\ 1, & j=1, \ldots, N-1 .\end{cases}
$$

Moreover, its higher derivative matrices may be expressed as

$$
\begin{aligned}
& \left(D_{N}^{m}\right)_{i j} \\
& =m\left[\left(D_{N}^{m-1}\right)_{i i}\left(D_{N}\right)_{i j}-\left(s_{i}-s_{j}\right)^{-1}\left(D_{N}^{m-1}\right)_{i j}\right], \\
& i \neq j, \\
& \left(D_{N}^{m}\right)_{i i}=-\sum_{j=0, j \neq i}^{N}\left(D_{N}^{m}\right)_{i j} .
\end{aligned}
$$

Thus, the differential operators in the governing equation and the BCs can be approximated by the differential matrix with the Chebyshev spectral approach. As an example, for a prismatic EB beam, the differential governing equation in (9) can be easily transformed to the following matrix eigenvalue problem:

$$
\left[A_{x}\right]^{-1}\left([J]\left[D_{N}^{4}\right]+2\left[J^{\prime}\right]\left[D_{N}^{3}\right]+\left[J^{\prime \prime}\right]\left[D_{N}^{2}\right]\right)\left\{\begin{array}{c}
\Phi_{0} \\
\Phi_{1} \\
\vdots \\
\Phi_{N}
\end{array}\right\}=\lambda\left\{\begin{array}{c}
\Phi_{0} \\
\Phi_{1} \\
\vdots \\
\Phi_{N}
\end{array}\right\},
$$

where $X_{i} \in[0,1]$ is a transformation of CGL collocation points $s_{j}, X_{i}=\left(s_{i}+1\right) / 2$, and

$$
[J]=\operatorname{diag}\left\{\begin{array}{c}
i\left(X_{0}\right) \\
i\left(X_{1}\right) \\
\vdots \\
i\left(X_{N}\right)
\end{array}\right\}
$$

$$
\left[J^{\prime}\right]=\operatorname{diag}\left\{\begin{array}{c}
i^{\prime}\left(X_{0}\right) \\
i^{\prime}\left(X_{1}\right) \\
\vdots \\
i^{\prime}\left(X_{N}\right)
\end{array}\right\} \text {, }
$$




$$
\begin{gathered}
{\left[J^{\prime \prime}\right]=\operatorname{diag}\left\{\begin{array}{c}
i^{\prime \prime}\left(X_{0}\right) \\
i^{\prime \prime}\left(X_{1}\right) \\
\vdots \\
i^{\prime \prime}\left(X_{N}\right)
\end{array}\right\},} \\
{\left[A_{x}\right]=\operatorname{diag}\left\{\begin{array}{c}
a\left(X_{0}\right) \\
a\left(X_{1}\right) \\
\vdots \\
a\left(X_{N}\right)
\end{array}\right\},} \\
\Phi_{i}=\Phi\left(X_{i}\right), \\
\lambda=\Omega^{4} .
\end{gathered}
$$

It should be noted that the LHS summed up matrices in (30) form the so-called system matrix of a nonprismatic EB beam.

\section{The Chebyshev Spectral Method for Boundary-Value Problems of an EB Beam}

There are two kinds of boundary conditions for the nonprismatic EB beam problem: one is the homogeneous Dirichlet boundary condition and the other is the boundary condition embedded with an eigenvalue, for example, tip-masses at both ends of the beam. The system matrix for the first kind boundary-value problem can be solved straightforwardly with a standard eigensolver. However, it is not easy to formulate the eigenvalue problem for the system matrix with the second kind boundary-value conditions imposed.

For the second kind boundary-value condition, we will briefly introduce the row replacement and the rectangular spectral collocation approaches in $[21,26]$ and present a detail null space approach to the corresponding boundary-value problem of an EB beam.

4.1. Row Replacement and Rectangular Spectral Collocation Approaches. From (9) to (13), one may obtain a generalized eigenproblem as follows:

$$
[D]_{N \times N}\{X\}_{N \times 1}=\lambda\{X\}_{N \times 1},
$$

where $\{X\}_{N \times 1}$ denotes the CGL collocation vector and $[D]_{N \times N}$ is the system matrix.

Vector $\{X\}$ can be decomposed as $\left\{X_{c}\right\},\left\{X_{\lambda}\right\}$, and $\left\{X_{r}\right\}$. $\left\{X_{c}\right\}$ is the collection of homogeneous boundary and interface conditions. $\left\{X_{\lambda}\right\}$ is the matrix of boundary and interface conditions containing the eigenvalues. $\left\{X_{r}\right\}$ is the residual matrix of the governing equation of an EB beam. Then, (32) can be partitioned as

$$
\left[\begin{array}{lll}
D_{r r} & D_{r c} & D_{r \lambda} \\
D_{c r} & D_{c c} & D_{c \lambda} \\
D_{\lambda r} & D_{\lambda c} & D_{\lambda \lambda}
\end{array}\right]_{N \times N}\left\{\begin{array}{l}
X_{r} \\
X_{c} \\
X_{\lambda}
\end{array}\right\}_{N \times 1}=\lambda\left\{\begin{array}{l}
X_{r} \\
X_{c} \\
X_{\lambda}
\end{array}\right\}_{N \times 1} .
$$

The homogeneous boundary conditions should satisfy the following equation:

$$
\left[\begin{array}{lll}
C_{c r} & C_{c c} & C_{c \lambda}
\end{array}\right]\{X\}=0 \cdot\left\{X_{c}\right\} .
$$

When boundary conditions include the eigenvalues, such as in (10) to (13), the solutions should satisfy the following equation:

$$
\left[\begin{array}{lll}
C_{\lambda r} & C_{\lambda c} & C_{\lambda \lambda}
\end{array}\right]\{X\}=\lambda \cdot\left\{X_{\lambda}\right\} .
$$

Substituting (34) and (35) into (33), by the row replacement approach we obtain the following expression:

$$
\left[\begin{array}{ccc}
D_{r r} & D_{r c} & D_{r \lambda} \\
C_{c r} & C_{c c} & C_{c \lambda} \\
D_{\lambda r}-C_{\lambda r} & D_{\lambda c}-C_{\lambda c} & D_{\lambda \lambda}-C_{\lambda \lambda}
\end{array}\right]\left\{\begin{array}{c}
X_{r} \\
X_{c} \\
X_{\lambda}
\end{array}\right\}=\lambda\left\{\begin{array}{c}
X_{r} \\
0 \\
0
\end{array}\right\} .
$$

Simplifying (36) by reducing the order of the matrix, we obtain the final expression with eigenvalues of boundary conditions:

$$
\left[D_{r r}-R_{r c}\left(R_{c c}\right)^{-1} R_{c r}\right]\left\{X_{r}\right\}=\lambda \cdot\left\{X_{r}\right\},
$$

where

$$
\begin{gathered}
{\left[R_{c c}\right]=\left[\begin{array}{cc}
C_{c c} & C_{c \lambda} \\
D_{\lambda c}-C_{\lambda c} & D_{\lambda \lambda}-C_{\lambda \lambda}
\end{array}\right],} \\
{\left[R_{c r}\right]=\left[\begin{array}{c}
C_{c r} \\
D_{\lambda r}-C_{\lambda r}
\end{array}\right],} \\
{\left[R_{c \lambda}\right]=\left[\begin{array}{ll}
R_{r c} & R_{c c}
\end{array}\right],} \\
{\left[R_{r c}\right]=\left[\begin{array}{ll}
D_{r c} & D_{r \lambda}
\end{array}\right] .}
\end{gathered}
$$

The row replacement method can be used to obtain the feasible eigensolutions for the well-conditioned system matrix after being reduced from the boundary conditions. However, for the classical two-point boundary-value problem, the eigensolutions can be achieved easier by removing the top and bottom rows of the differential matrix. However, for EB beams with interface jump conditions, row replacement can become rather complicated in building up the spectral collocation matrix in the LHS of (36). Moreover, the row replacement method does not always produce eigenvalues that have the same qualitative properties, such as symmetry, as the original operator. Thus, the matrix in (37), even for the simplest differential equation, is full and ill-conditioned, so it is in general not advisable to solve (37) using a direct method for large $N$. Instead, some appropriate preconditioning approach should be used in [16].

Driscoll and Hale [26] propose a rectangular spectral approach, based upon resampling the differentiated polynomials into a lower-degree subspace to make the differentiation matrices. The rectangular collocation approach is described as follows:

(a) The square system matrix $(N \times N)$ originally constructed according to the CSM is allocated for $N+m$ columns by barycentric resampling discretization and expanded to a rectangular matrix $(N \times[N+m]$, where $m$ is the number of boundary conditions). 
(b) The $m$ sets of boundary conditions formed by CSM are then placed above the rectangular matrix and reconstruct a square $[D]_{(N+m) \times(N+m)}$ matrix for eigensolver.

This approach avoids ambiguities that arise when applying the classical row deletion method in boundary-value problems. However, as indicated in [28], it shows several orders of magnitude less accuracy when evaluated using the resampling points, but higher accuracy when evaluated directly at the original collocation points.

4.2. Projection by Using the Null Space Approach. In this paper, we propose a novel null space approach to solve the eigenvalue problems of the system matrix by preserving its original dimension and characteristics. According to the definition, by (33) we have $\left[D_{s}\right] \in \mathbb{R}^{N \times N},\{X\} \in \mathbb{R}^{N \times 1}$, and matrix $\left[C_{s}\right] \in \mathbb{R}^{m \times N}$ represents the imposed boundary and interface conditions. The rank of matrices $\left[D_{s}\right]$ and $\left[C_{s}\right]$ is $N$ and $m(N>m)$, respectively. The eigenproblem of (33) must satisfy the requirement, $\left[C_{s}\right]\{X\}=\{0\}$. Let the null space of $\left[C_{s}\right]$ be matrix $\left[N_{c}\right]$ and satisfy the identity $\left[C_{s}\right]\left[N_{c}\right]=[0]$. It should be noted that $\{X\}$ is a vector in physical space, and it may be projected to vector $\{\xi\}$ in the null space with matrix $\left[N_{c}\right]$; i.e., $\{X\}=\left[N_{c}\right]\{\xi\}$. As a result, (33) may be projected to the null space of $\left[C_{s}\right]$ as follows:

$$
\left[D_{s}\right]\left[N_{c}\right]\{\xi\}=\lambda\left[N_{c}\right]\{\xi\} \text {. }
$$

Premultiplying both sides of (39) with the transposed $\left[N_{c}\right]$, and taking advantage of the orthonormal property in $\left[N_{c}\right]$, we get

$$
\begin{gathered}
{\left[N_{c}\right]^{T}\left[D_{s}\right]\left[N_{c}\right]\{\xi\}=\lambda\left[N_{c}\right]^{T}\left[N_{c}\right]\{\xi\}=\lambda\{\xi\},} \\
{\left[D_{s}^{\nu}\right]\{\xi\}=\lambda\{\xi\},} \\
{\left[D_{s}^{v}\right]=\left[N_{c}\right]^{T}\left[D_{s}\right]\left[N_{c}\right] .}
\end{gathered}
$$

Consequently, (33) is reduced to (40) by projecting the system matrix of the physical space to the null space which is imposed by the boundary and interface conditions. For $\left\{\xi_{\lambda}\right\}$, an eigenvector in (40), one may restore the eigenvector in physical space by setting $\left\{X_{\lambda}\right\}=\left[N_{c}\right]\left\{\xi_{\lambda}\right\}$.

The null space approach may actually improve the matrix condition of EB beams. For problems with large variations or consisting of independent rows or columns in the elements of matrix $\left[D_{s}\right]$, a robust null space preconditioning approach will improve the potential ill-conditioned problems, such as the system matrix formulated from a partially clamped EB beam, and overcome the numerical issues in the modal solutions that include both high and low order modes.

4.3. Roadmap for EB Beam Eigenvalue Problems with the Aid of the Chebfun Toolbox. In this section, we discuss the solution roadmap by using the Chebfun toolbox and deal with EB beams with various boundary and interface conditions. The Chebf un toolbox in MATLAB environment is developed by Professor L. N. Trefethen in the Cambridge University
$[24,25]$. This toolbox is a collection of commands based on a Chebyshev expansion algorithm, where the number of expansion terms is controlled by mechanical precision. The chebop utility in the Chebfun toolbox enables a symbolic operator syntax to be applied to numerical objects and implements the recasting process of governing equations of differential equations. In addition, this toolbox is constructed in MATLAB by matrices and vectors and uses automatic Chebyshev polynomial interpolation to represent functions and automatic spectral collocation methods to approximate operators. With the MATLAB built-in numerical functions of the toolbox, one may quickly and accurately get the asymptotic solutions of various problems.

As an illustrated example, Figure 3 is a solution roadmap for a partially clamped EB beam (see (16) and (17)). In the first step, the governing equation of the EB beam with partially clamped boundary conditions is constructed by using the chebop syntax. Through the chebop system, one may easily define the corresponding EB beam governing equation as well as the left and right boundary conditions (L . op, L . Ibc, and L.rbc) and convert them into the Chebyshev spectral matrix in the MATLAB working space. Next, it shows the null space approach for building the orthonormal space of the boundary conditions (nuA) and the projection of the governing differential matrix into the corresponding null space (Aend) thus obtaining the solution with the MATLAB builtin eigensolver. The last step is projecting the eigenvectors backward into the physical space $(\mathrm{Vx})$, which represents the physical modal shapes of the EB beam.

4.4. Modal Analysis of a Nonprismatic Tip-Massed EB Beam with Partially Clamped Boundary Conditions. We demonstrate how to use CSM together with NSA through the Chebf un toolbox to solve the nonprismatic EB beam that tipmassed and elastically restrained at both ends.

The governing equation in (9) may be expressed with differential operators as follows:

$$
\Omega^{4} \Phi(X)=F_{0}(X, D) \Phi(X)
$$

where $F_{0}(X, D)=i^{\prime \prime}(X) D^{2}+2 i^{\prime}(X) D^{3}+i(X) D^{4}, D^{k} \Phi(X)=$ $d^{k} \Phi(X) / d x^{k}$.

The derivative of (41) with respect to $X$ is

$$
\Omega^{4} \Phi^{\prime}(X)=F_{1}(X, D) \Phi(X)
$$

where $F_{1}(X, D)=i^{\prime \prime \prime}(X) D^{2}+3 i^{\prime \prime}(X) D^{3}+3 i^{\prime}(X) D^{4}+i(X) D^{5}$.

For boundary conditions (10)-(13), all the terms containing $\Omega^{4}$ can be removed by substituting the RHSs of (41) and (42), together with a simple manipulation to get

$$
\begin{aligned}
& \Phi^{\prime \prime}(0)-K_{R L} \Phi^{\prime}(0) \\
& \quad+\mu_{m L}\left[\left(\delta_{L}^{2}+\gamma_{L}^{2}\right) F_{1}(0, D)-\delta_{L} F_{0}(0, D)\right] \Phi(0) \\
& \quad=0
\end{aligned}
$$




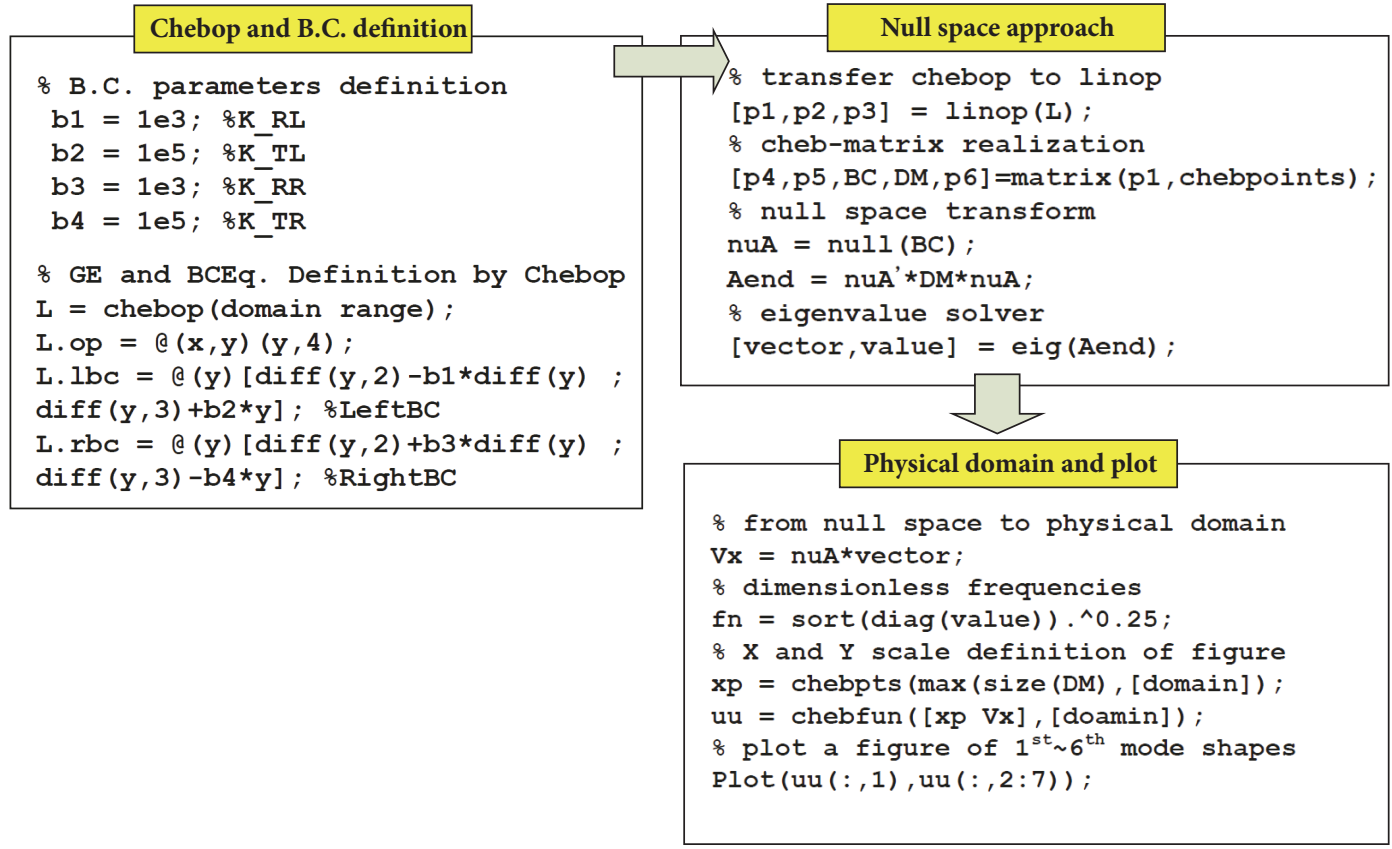

FIGURE 3: The MATLAB scripts and procedures of CSM with NSA.

$$
\begin{aligned}
& \Phi^{\prime \prime \prime}(0)-i^{\prime}(0) \Phi^{\prime \prime}(0) \\
& +\left\{\mu_{m L}\left[\delta_{L} F_{1}(0, D)-F_{0}(0, D)\right]+K_{T L}\right\} \Phi(0)=0 \\
& \Phi^{\prime \prime}(1)+K_{R R} \Phi^{\prime}(1) \\
& \quad-\mu_{m R}\left[\left(\delta_{R}^{2}+\gamma_{R}^{2}\right) F_{1}(1, D)+\delta_{R} F_{0}(1, D)\right] \Phi(1) \\
& \quad=0, \\
& \Phi^{\prime \prime \prime}(1)-\frac{i^{\prime}(1)}{i(1)} \Phi^{\prime \prime}(1) \\
& +\left\{\mu_{m R}\left[\delta_{R} F_{1}(1, D)+F_{0}(1, D)\right]-K_{T R}\right\} \Phi(1) \\
& =0 .
\end{aligned}
$$

After that, one may follow the roadmap in Figure 3, where the boundary conditions (43) to (46) can be converted to the $\left[C_{s}\right](4 \times N)$ matrix by using $N$-points CSM, and we get the corresponding $(N-4) \times N$ matrix of the null space. With the NSA (see (39)-(40)) applied, the modal solutions can then be obtained by using a standard eigensolver.

4.5. Modal Analysis of Stepped EB Beams. For a stepped EB beam, (16) and (17) form the governing equation and interface conditions for each segment, and (18) is the boundary condition at both ends. Equations (16) and (18) can be defined with the help of the chebop system as shown in the previous section. According to the $R_{j}$ ratio at each segment, (16) may be transformed into a Chebfun object by multiplied with the Heaviside function:

$$
\left[1+\left(\mu_{j}-1\right) \cdot \text { Heaviside }\left(X_{R j}\right)\right]^{-1} D^{4} \Phi=\Omega^{4} \Phi .
$$

On the other hand, the LHS of (18) can be expressed using a differential operator notation as

$$
\begin{aligned}
D^{2} \Phi(0)-K_{R L} D \Phi(0) & =0, \\
D^{3} \Phi(0)+K_{T L} \Phi(0) & =0,
\end{aligned}
$$

while the LHS of (18) can be rewritten using the differential operator notation by

$$
\begin{gathered}
\eta_{N-1} D^{2} \Phi(1)+K_{R R} D \Phi(1)=0, \\
\eta_{N-1} D^{3} \Phi(1)-K_{T R} \Phi(1)=0 .
\end{gathered}
$$

Furthermore, in (17), the displacement compatibility (identical jump conditions) and force equilibrium (scaled jump conditions) at the interface of each section can be established by using the jump command in chebop system, as follows:

$$
\begin{aligned}
& \text { [jump }\left(\Phi, R_{j}\right) \text {; jump }\left(D \Phi, R_{j}\right) \text {; jump }\left(D^{2} \Phi, R_{j}\right) \text {; jump } \\
& \left.\left(D^{3} \Phi, R_{j}\right)\right] \text {. }
\end{aligned}
$$

Since the jump command in the chebop system can only handle the identical jump conditions but is unable to exactly convert the scaled jump conditions of the stepped beam, extra manipulations on the rows of the interface conditions in the differential matrix are needed to define the scaled jump conditions.

For example, a two-step EB beam is shown in Figure 4. With ten CGL collocated points for each beam segment, its differential matrix should consist of 30 rows and columns. In that matrix, the first four rows are formulated using the boundary conditions of both ends, while the fifth to twelfth rows are formulated using the displacement compatibility (identical jump) conditions and force equilibrium (scaled 


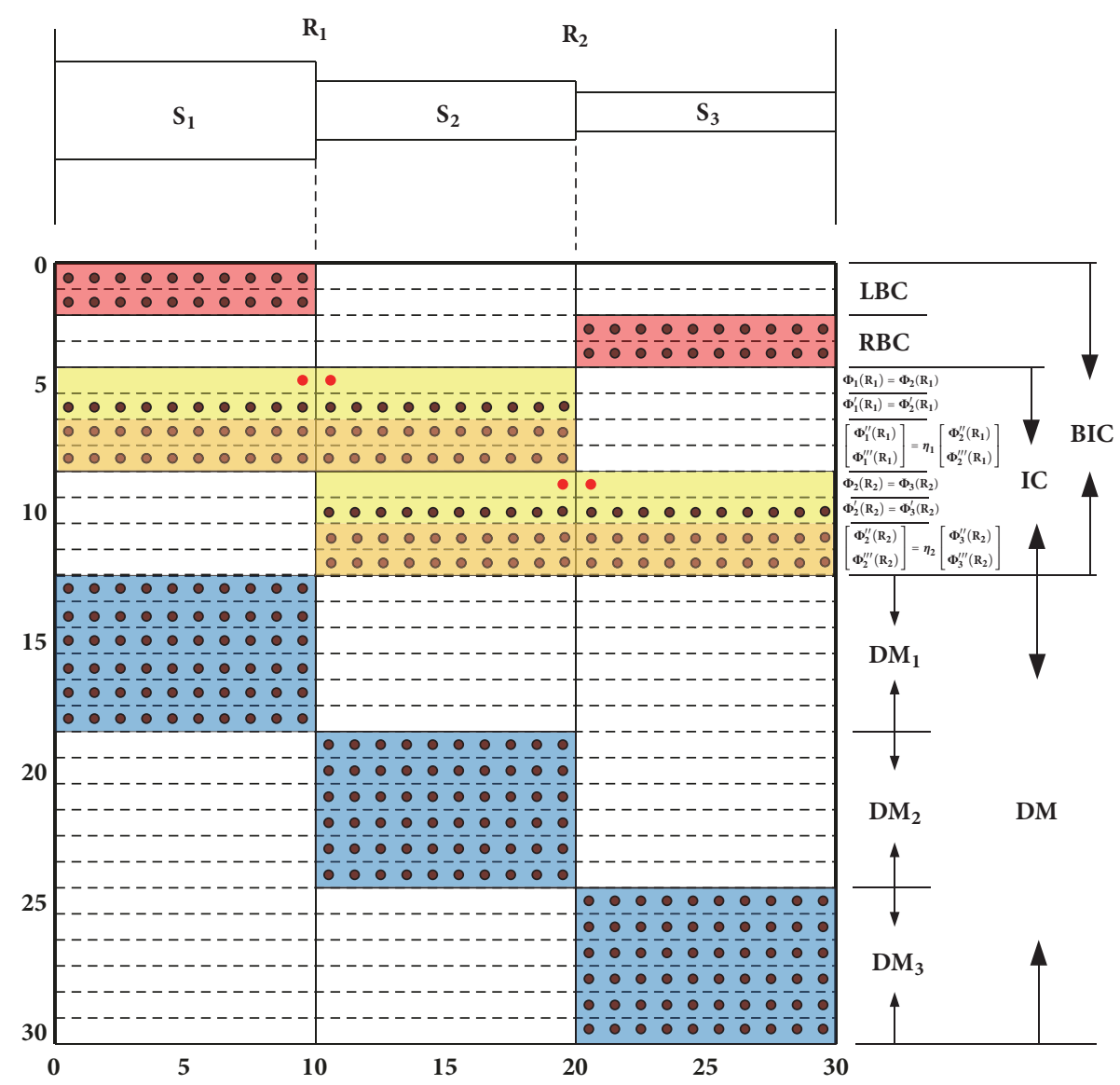

FIgURE 4: The CSM differential matrix with ten CGL collocated points for three-steps EB beam.

jump) conditions at the interface of the steps. It should be noted that the scaled jump conditions, appearing on rows $7,8,11$, and 12 , should be modified with the scale $\eta_{j}$ in (17). The last 18 rows (the DM part in Figure 4) are the governing equation of each beam segment stacked up diagonally with each corresponding differential matrix. After that, the corresponding null space matrix is obtained by using these first 12-row partition (the BIC part in Figure 4). With the NSA (see (39) and (40)) applied, the modal solutions can be obtained by using a standard eigensolver.

\section{Numerical Calculations}

In order to verify the proposed CSM with NSA approach for analyzing the free vibration of EB beam step by step, four numerical examples with different beam styles and boundary conditions are discussed in this section. All results were obtained using MATLAB R2013B and Chebfun 5.1 toolbox on a HP EliteDesk with an Intel Core i5-6500MQ @ $3.30 \mathrm{GHz}$ CPU and 4 GB RAM.

5.1. Simply Supported Prismatic EB Beam. The exact modal solution of a simply supported prismatic EB beam is used for validating the accuracy of the proposed CSM with NSA approach as well as the CSM with RSC (resampling spectral collocation) approach. For a simply supported prismatic EB beam, the exact nondimensional modal frequencies are $n \pi$, and the corresponding mode shapes are $\sin (n \pi)$, respectively, where $n$ is the mode order.

With the Chebfun toolbox, one may use the chebop system to define the governing equation and boundary conditions and can obtain the eigensolution using the overloaded eigs command in the Chebfun toolbox. It should be noted that this eigensolution is based on the RSC method built in the Chebfun toolbox. The proposed CSM with NSA approach is illustrated in Figure 3, which projects the differential matrix from the EB beam to the null space of the boundary conditions. In order to benchmark the accuracy of the eigensolutions with these two approaches, the natural frequencies of the 15th and 100th mode are solved separately.

Table 1 compares the exact natural frequencies with the RSC and present approach (CSM with NSA) for a simply supported EB beam. The selected CGL collocated points for the differential matrices are $N=30$ and $N=200$, respectively. However, in accordance with the sampling theorem, the maximum number of modes $\left(n_{M}\right)$ should not be larger than $N / 2$ in order to get rid of aliasing. In Table 1, we display the cases of $n_{M}=10$ and $n_{M}=100$ only. As the results indicate, the accuracy of the present approach outperforms the RSC approach in both cases. However, for the RSC approach with $N=200$, results are unclear since the lower-order modes are distorted. We conjecture that the resampling algorithm of 
TABLE 1: Comparison of dimensionless natural frequencies for $n_{M}=10$ and $n_{M}=100$ by exact solutions, RSC, and present methods.

\begin{tabular}{|c|c|c|c|c|c|c|c|c|c|}
\hline CGL points & $n_{M}$ & Method & $\Omega_{1}$ & $\Omega_{2}$ & $\Omega_{3}$ & $\cdots$ & $\Omega_{n_{M^{-2}}}$ & $\Omega_{n_{M^{-1}}}$ & $\Omega_{n_{M}}$ \\
\hline \multirow{3}{*}{30} & \multirow{3}{*}{10} & Exact & 3.14159 & 6.28319 & 9.42478 & $\cdots$ & 40.84070 & 43.98230 & 47.12389 \\
\hline & & RSC & 3.14076 & 6.29299 & 9.42456 & $\ldots$ & 40.84072 & 43.98240 & 47.12414 \\
\hline & & Present & 3.14159 & 6.28319 & 9.42478 & $\ldots$ & 40.84070 & 43.98225 & 47.12390 \\
\hline \multirow{3}{*}{200} & \multirow{3}{*}{100} & Exact & 3.14159 & 6.28319 & 9.42478 & $\ldots$ & 307.87608 & 311.01767 & 314.15927 \\
\hline & & RSC & $3.709444+(3.70944 i)$ & 8.96197 & 9.05268 & $\cdots$ & 307.87394 & 311.04696 & 314.16051 \\
\hline & & Present & 3.14159 & 6.28319 & 9.42478 & $\ldots$ & 307.87608 & 311.01767 & 314.15927 \\
\hline
\end{tabular}

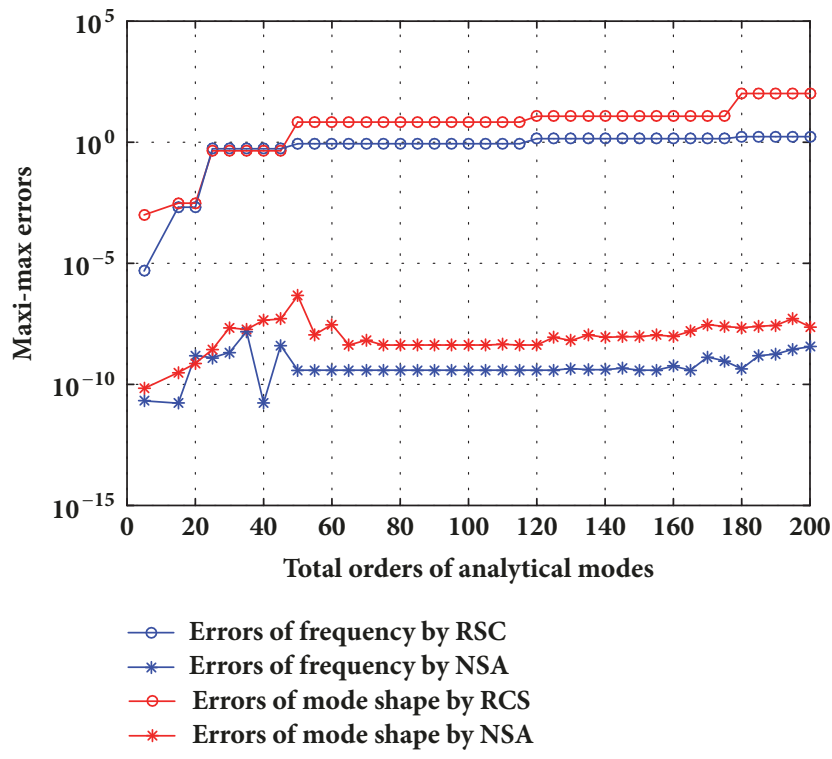

FIGURE 5: Normalized maxi-max errors of frequencies and mode shapes by using RSC and NSA approaches.

RSC goes unstable and oversample the lower-order modes as claimed in [28]. Consequently, we suggest the following rule of thumb for the present approach to select the number of CGL points:

$$
\begin{aligned}
\text { if } n_{M} & <11, \\
N & =3 n_{M} ; \\
\text { else, } N & =2 n_{M} .
\end{aligned}
$$

The eigenvectors (mode shapes) solved by these two approaches can be compared to the exact solution, and we obtain the normalized maxi-max error as follows:

$$
\begin{aligned}
& E_{\mathrm{RSC}}=\max _{n}\left|1-\frac{\int_{0}^{l} \phi_{n}^{2}(x)_{\mathrm{RSC}} d x}{\int_{0}^{l} \phi_{n}^{2}(x)_{\mathrm{exact}} d x}\right|, \\
& E_{\mathrm{NSA}}=\max _{n}\left|1-\frac{\int_{0}^{l} \phi_{n}^{2}(x)_{\mathrm{NSA}} d x}{\int_{0}^{l} \phi_{n}^{2}(x)_{\text {exact }} d x}\right|, \quad n=1,2, \ldots, 5 .
\end{aligned}
$$

Following the criteria in (50) for selecting number of CGL collocated points and the maximum number of modes $\left(n_{M}\right)$, Figure 5 and Table 2 show the normalized maxi-max errors of the natural frequencies and mode shapes in the first five modes, analyzed by using the RSC and NSA approaches. It is clear that the accuracy of the modal solutions by using the NSA approach outperforms the RSC approach at all chosen $n_{M}$.

5.2. Higher-Order Modal Solutions for a Partially Clamped Prismatic EB Beam. The partially clamped conditions of an EB beam are defined by four elastic restrained coefficients $\beta_{1}$, $\beta_{2}, \beta_{3}$, and $\beta_{4}$ in (22). The higher-order asymptotic modal solution, obtained in (25), is denoted by $\mathrm{PC}_{1}$. Moreover, $\mathrm{PC}_{2}$ denotes the null space approach (NSA) as shown in Figure 3 for dealing with the eigenproblem of a partially clamped prismatic EB beam. The exact modal solution of a partially clamped uniform EB beam is not found in the literature, to the best of our knowledge. The modal solutions with classical boundary conditions (clamped (C), free (F), pinned $(\mathrm{P})$, slider (S), and simply supported (S-S)) are adopted as a baseline and denoted by $\mathrm{PC}_{0}$, such as the asymptotic solution proposed in [8] for higher-order modal solutions.

The asymptotic magnitude of the elastic coefficients for free and fixed conditions of a partially clamped beam is around 0 and $10^{8}$, respectively. $\mathrm{PC}_{1}$ is substituted in combination with the asymptotic free and fixed $\beta_{i}$ into (25). In Figure 3, $\mathrm{PC}_{2}$ uses the Chebf un toolbox to solve the modal solutions with the asymptotic magnitudes of $\beta_{i}$. For $\mathrm{PC}_{0}$, we use the classical boundary conditions as proposed in [8]. With the classical C-C, C-F, C-S, and C-P boundary conditions, the modal solutions for the first 200 modes of the EB beam are obtained by higher-order asymptotic analysis of the partial-clamped solutions $\left(\mathrm{PC}_{1}\right)$ and the NSA approach $\left(\mathrm{PC}_{2}\right)$. Then, these results are compared to the asymptotic modal solutions as proposed in [8] $\left(\mathrm{PC}_{0}\right)$. The normalized maxi-max deviations of the natural frequency between $\mathrm{PC}_{1}$, $\mathrm{PC}_{2}$, and $\mathrm{PC}_{0}$ are defined by

$$
\begin{aligned}
& e_{\mathrm{PC} 1}=\max _{n}\left|1-\frac{\Omega_{n, P C 1}}{\Omega_{n, P C 0}}\right|, \\
& e_{\mathrm{PC} 2}=\max _{n}\left|1-\frac{\Omega_{n, P C 2}}{\Omega_{n, P C 0}}\right|,
\end{aligned}
$$

and the normalized maxi-max deviations of the mode shape between $\mathrm{PC}_{1}, \mathrm{PC}_{2}$, and $\mathrm{PC}_{0}$ are defined by

$$
E_{\mathrm{PC} 1}=\max _{n}\left|1-\frac{\int_{0}^{l} \phi_{n}^{2}(x)_{\mathrm{PC} 1} d x}{\int_{0}^{l} \phi_{n}^{2}(x)_{\mathrm{PC} 0} d x}\right|,
$$


TABLE 2: Maxi-max errors of natural frequencies and mode shapes by using RSC and NSA approaches.

\begin{tabular}{|c|c|c|c|c|}
\hline \multirow{2}{*}{ Mode order } & \multicolumn{2}{|c|}{ Maxi-max errors of natural frequencies } & \multicolumn{2}{|c|}{ Maxi-max errors of mode shapes } \\
\hline & RSC & NSA & RSC & NSA \\
\hline 5 & $2.73 \mathrm{E}-06$ & $2.09 \mathrm{E}-11$ & $1.39 \mathrm{E}-06$ & $6.77 \mathrm{E}-11$ \\
\hline 10 & $1.69 \mathrm{E}-03$ & $2.79 \mathrm{E}-11$ & $1.44 \mathrm{E}-02$ & 3.33E-11 \\
\hline 15 to 20 & 0.001685759 & $<1.56 \mathrm{E}-09$ & 0.014375 & $<7.33 \mathrm{E}-10$ \\
\hline 25 to 45 & 0.556364694 & $<1.53 \mathrm{E}-08$ & 0.332986 & $<5.01 \mathrm{E}-08$ \\
\hline 30 to 45 & 0.556364694 & 2.09E-09 & 0.332986 & $2.17 \mathrm{E}-08$ \\
\hline 50 to 110 & 1.046748562 & $3.86 \mathrm{E}-10$ & 0.865779 & $<4.59 \mathrm{E}-07$ \\
\hline 115 to 170 & 1.755796169 & $<1.35 \mathrm{E}-09$ & 0.931819 & $<2.96 \mathrm{E}-08$ \\
\hline 175 to 200 & 0.909712404 & $<3.59 \mathrm{E}-09$ & 0.994279 & $<5.22 \mathrm{E}-08$ \\
\hline
\end{tabular}

$$
E_{\mathrm{PC} 2}=\max _{n}\left|1-\frac{\int_{0}^{l} \phi_{n}^{2}(x)_{\mathrm{PC} 2} d x}{\int_{0}^{l} \phi_{n}^{2}(x)_{\mathrm{PC} 0} d x}\right|
$$

The maxi-max deviations of the modal solutions at the specified modes of order (10:10:100 and 125:25:200) are shown in Figures 6 and 7 and Table 3, respectively. As we can see, both $\mathrm{PC}_{1}$ and $\mathrm{PC}_{2}$ approaches attain around the same accuracy level. In this case, they are in excellent agreement with the modal solution of both approaches and this encourages us to conclude the following:

(a) The result from $\mathrm{PC}_{1}$ validates the asymptotic higherorder modal solutions of a partial-clamped beam in the Appendix and avoids the numerical problems that arise at higher-order modes.

(b) The accuracy of the proposed CSM with NSA approach is nearly equal to the asymptotic analytical solution. This approach offers a robust and systematic procedure without going through sophisticated symbolic derivations needed for the EB beam with a variety of boundary conditions.

5.3. Modal Solution for a Partially Clamped and Tip-Massed Nonprismatic EB Beam. In this example, we attempt to validate the robustness of our proposed approach for a tip-massed nonprismatic EB beam with a variety of BCs. It should be noted that, for a tip-massed EB beam, the boundary conditions are embedded in the eigenvalue to be solved. The governing equation (9) and BCs (see (10)-(12)) are formulated with the Chebfun toolbox and the chebop system through (42)-(46) in Section 4.4. Then the modal solutions are obtained with the NSA projection and solved by the MATLAB eig command. However, for comparison with [11], the BCs of the beam are tip-massed at the left end and fixed at the right end $\left(\beta_{3} \longrightarrow \infty, \beta_{4} \longrightarrow \infty\right)$, while the other relevant beam parameters have the same value as those in [11] $\left(\gamma_{L}=0,0.3\right.$ and $0.6, \alpha=1.2$ and $2 ; \mu_{L}=0.2$ and 2$)$. Finally, the lowest three natural frequencies and associated mode shapes of a tip-massed nonprismatic EB beam with various BCs are evaluated and compared with [11].

Table 4 shows the first three nondimensional natural frequencies of a left-end tip-massed cantilever cone beam by

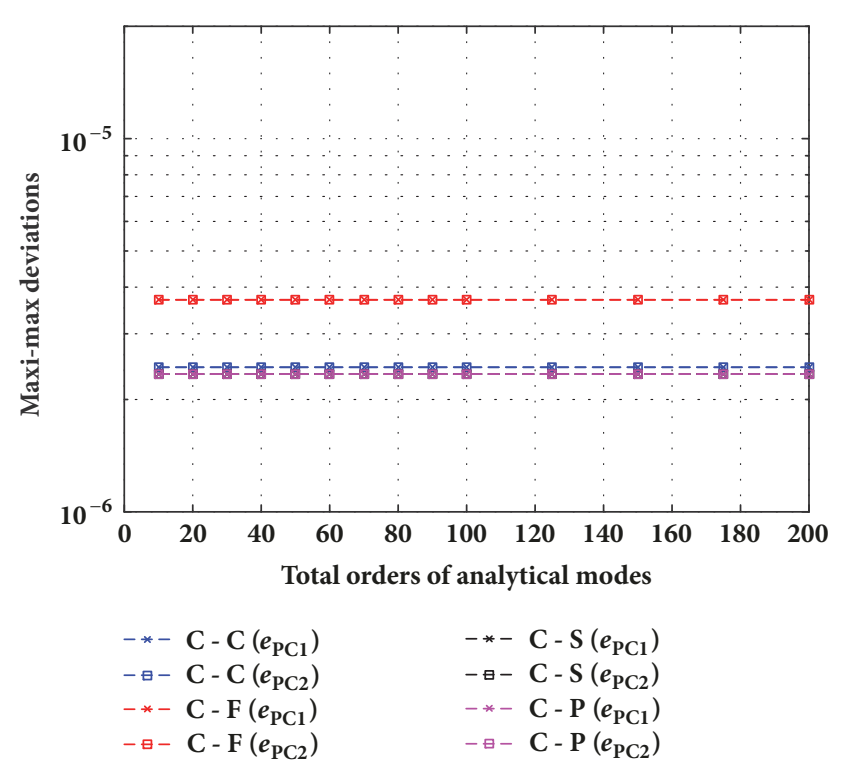

FIGURE 6: Normalized maxi-max deviations of natural frequency by using $\mathrm{PC} 1$ and $\mathrm{PC} 2\left(e_{\mathrm{PC} 1}\right.$ and $\left.e_{\mathrm{PC} 2}\right)$.

using the proposed approach and compares the results with [11]. Using different taper ratio, left-end tip-mass, and tipmass rotatory inertia (i.e., $\beta_{1}=0, \beta_{2}=0, \beta_{3} \longrightarrow \infty, \beta_{4} \longrightarrow \infty$, $\mu_{R}=\delta_{R}=\gamma_{R}=\delta_{L}=0, \gamma_{L}=0$ and $0.6, \alpha=1.2$ and 2 , and $\mu_{L}=$ 0.2 and 2), the accuracy of the natural frequencies competes with [11] up to 3 decimal points, with the corresponding mode shapes shown in Figure 8. Moreover, Table 5 and Figures 9-11 display the results for a cantilever cone beam with the effects of tip-mass rotatory inertia and eccentricity at the left end, (i.e., $\alpha_{b}=\alpha_{h}=\alpha=1.1, \beta_{1}=0, \beta_{2}=0, \mu_{R}=\delta_{R}=\gamma_{R}=\mu_{L}=0, \delta_{L}$ $=0.4$ and $0.6, \beta_{3}=0.1,1.0$ and 10 , and $\beta_{4}=1$ and $\infty$ ), and we obtain the same accuracy level as in the previous case. Consequently, for this example of a nonprismatic EB beam with eigenvalue embedded BCs, the robustness and accuracy of the proposed approach can be verified by the numerical results that compare favorably to [11].

5.4. Modal Analysis of a Uniform Stepped EB Beam with Classical Boundary Conditions. In order to validate the proposed method for the modal analysis of a stepped beam, here we 
TABLE 3: Normalized maxi-max deviations of modal solutions for $\mathrm{PC}_{1}$ and $\mathrm{PC}_{2}$ with four classical $\mathrm{BCs}$ imposed.

\begin{tabular}{lccccc}
\hline $\begin{array}{l}\text { Boundary } \\
\text { condition }\end{array}$ & Mode order & \multicolumn{2}{c}{ Maxi-max deviations of natural frequency } & \multicolumn{2}{c}{ Maxi-max deviations of mode shape } \\
\hline C-C & 10 to 200 & $2.44 \mathrm{E}-06$ & $2.44 \mathrm{E}-06$ & 0.001926 & $\mathrm{PC}_{2}$ \\
\hline C-F & 10 to 90 & $3.70 \mathrm{E}-06$ & $3.70 \mathrm{E}-06$ & 0.001014 & $3.70 \mathrm{E}-06$ \\
& 100 to 200 & $3.70 \mathrm{E}-06$ & $3.70 \mathrm{E}-06$ & 0.001014 & $2.84 \mathrm{E}-06$ \\
\hline & 10 to 50 & $2.34 \mathrm{E}-06$ & $2.34 \mathrm{E}-06$ & $7.35 \mathrm{E}-06$ & $2.89 \mathrm{E}-04$ \\
\hline C-P & 60 to 80 & $2.34 \mathrm{E}-06$ & $2.34 \mathrm{E}-06$ & $8.49 \mathrm{E}-06$ & $7.35 \mathrm{E}-06$ \\
& 90 to 100 & $2.34 \mathrm{E}-06$ & $2.34 \mathrm{E}-06$ & $<1.04 \mathrm{E}-04$ & $8.49 \mathrm{E}-06$ \\
\hline C-S & 125 to 200 & $2.34 \mathrm{E}-06$ & $2.34 \mathrm{E}-06$ & 0.075393 & $<1.04 \mathrm{E}-04$ \\
\hline
\end{tabular}

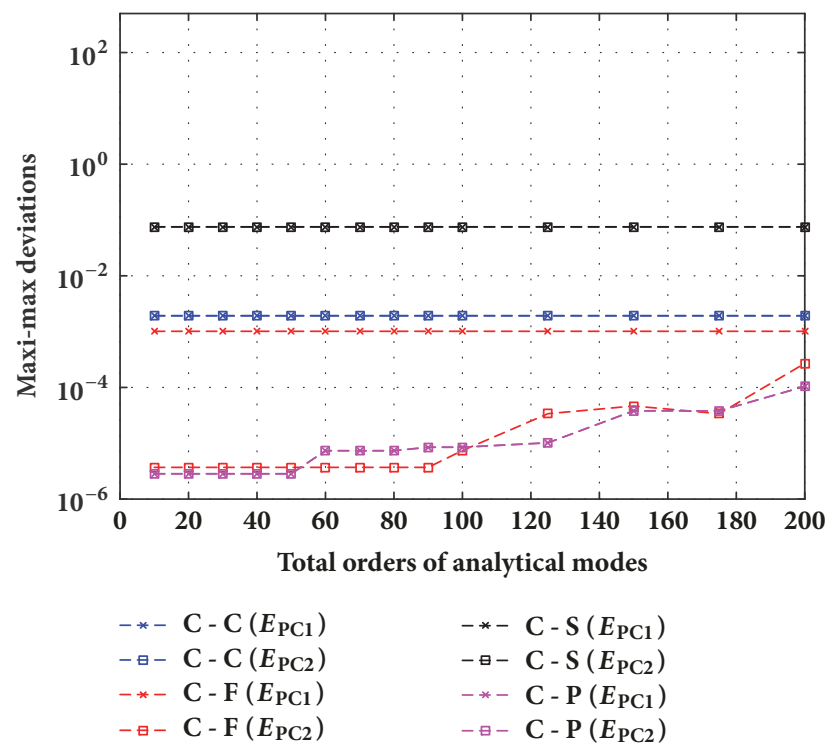

Figure 7: Normalized maxi-max deviations of mode shape by using PC1 and PC2 ( $E_{\mathrm{PC} 1}$ and $\left.E_{\mathrm{PC} 2}\right)$.

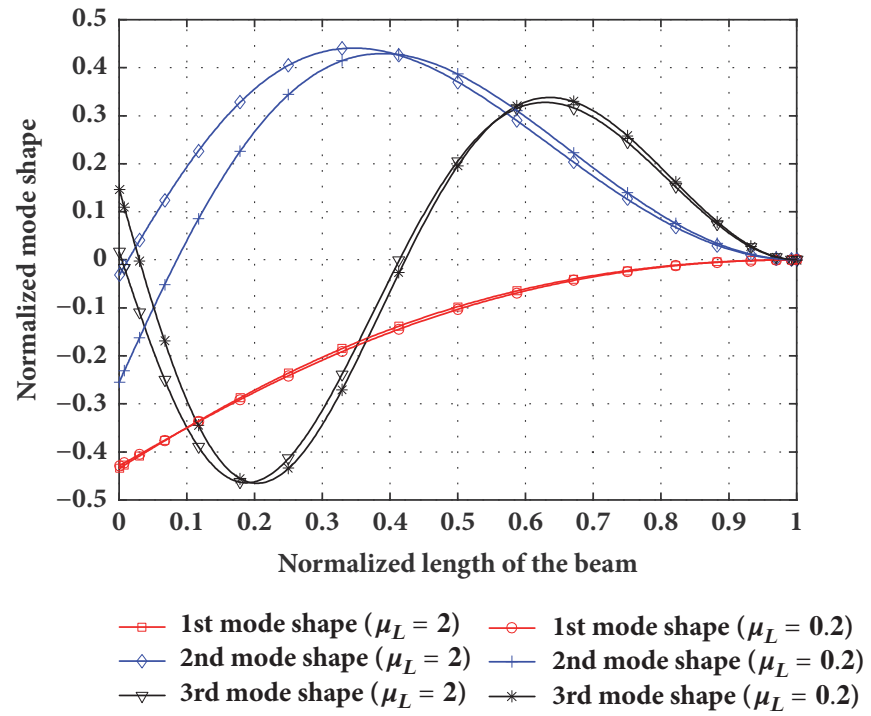

FIGURE 8: The first three normalized mode shapes of a cantilever cone beam with different left tip-mass ratio $\left(\mu_{L}\right)$. 
TABLE 4: The first three dimensionless natural frequencies of a cantilever cone beam with tip-mass, the rotatory inertia of mass, and its eccentricity at the left end $\left(\beta_{1}=0, \beta_{2}=0, \beta_{3} \longrightarrow \infty, \beta_{4} \longrightarrow \infty\right.$, and $\left.\mu_{R}=\delta_{R}=\gamma_{R}=\delta_{L}=0\right)$.

\begin{tabular}{|c|c|c|c|c|c|c|c|c|}
\hline \multirow{2}{*}{$\gamma_{L}$} & \multirow{2}{*}{$\alpha$} & \multirow{2}{*}{$\mu_{L}$} & \multicolumn{2}{|c|}{$\Omega_{1}$} & \multicolumn{2}{|c|}{$\Omega_{2}$} & \multicolumn{2}{|c|}{$\Omega_{3}$} \\
\hline & & & Present & (Ref. [7]) & Present & (Ref. [7]) & Present & (Ref. [7]) \\
\hline \multirow[t]{4}{*}{0.0} & 1.2 & 0.2 & 1.805113 & 1.805116 & 4.531398 & 4.531399 & 7.682832 & 7.682833 \\
\hline & & 2.0 & 1.180606 & 1.180605 & 4.242896 & 4.242897 & 7.478641 & 7.478641 \\
\hline & 2.0 & 0.2 & 2.392504 & 2.392498 & 5.375541 & 5.375538 & 8.914098 & 8.914100 \\
\hline & & 2.0 & 1.485717 & 1.485704 & 5.108411 & 5.108415 & 8.760282 & 8.760286 \\
\hline \multirow[t]{4}{*}{0.3} & 1.2 & 0.2 & 1.759466 & 1.759470 & 3.493511 & 3.493510 & 5.666955 & 5.666955 \\
\hline & & 2.0 & 1.122790 & 1.122787 & 2.228089 & 2.228088 & 5.079316 & 5.079316 \\
\hline & 2.0 & 0.2 & 2.268671 & 2.268663 & 3.648412 & 3.648385 & 6.290178 & 6.290136 \\
\hline & & 2.0 & 1.363150 & 1.363162 & 2.253308 & 2.253207 & 5.818125 & 5.818075 \\
\hline \multirow[t]{4}{*}{0.6} & 1.2 & 0.2 & 1.634321 & 1.634324 & 2.779848 & 2.779846 & 5.472033 & 5.472032 \\
\hline & & 2.0 & 0.991768 & 0.991773 & 1.795564 & 1.795562 & 5.051224 & 5.051225 \\
\hline & 2.0 & 0.2 & 1.954460 & 1.954460 & 3.049005 & 3.048977 & 6.199510 & 6.199473 \\
\hline & & 2.0 & 1.124889 & 1.124886 & 1.935342 & 1.935209 & 5.807056 & 5.807008 \\
\hline
\end{tabular}

TABLE 5: The first three dimensionless natural frequencies of a cone beam with tip-mass, the rotatory inertia of mass, and its eccentricity at the left end $\left(\alpha_{b}=\alpha_{h}=\alpha=1.1, \beta_{1}=0, \beta_{2}=0\right.$, and $\left.\mu_{R}=\delta_{R}=\gamma_{R}=\delta_{L}=0\right)$.

\begin{tabular}{|c|c|c|c|c|c|c|c|c|c|}
\hline \multirow{2}{*}{$\delta_{L}$} & \multirow{2}{*}{$\beta_{4}$} & \multirow{2}{*}{$\gamma_{L}$} & \multirow{2}{*}{$\beta_{3}$} & \multicolumn{2}{|c|}{$\Omega_{1}$} & \multicolumn{2}{|c|}{$\Omega_{2}$} & \multicolumn{2}{|c|}{$\Omega_{3}$} \\
\hline & & & & Present & (Ref. [7]) & Present & (Ref. [7]) & Present & (Ref. [7]) \\
\hline \multirow[t]{9}{*}{0.4} & $\infty$ & 0.6 & 0.1 & 0.467442 & 0.467440 & 1.854357 & 1.854354 & 4.358113 & 4.358113 \\
\hline & & & 1.0 & 0.755242 & 0.755247 & 1.948854 & 1.948854 & 4.454283 & 4.454283 \\
\hline & & & 10.0 & 0.934564 & 0.934568 & 2.182581 & 2.182581 & 4.834589 & 4.834589 \\
\hline & & 1.0 & 0.1 & 0.441666 & 0.441668 & 1.583464 & 1.583463 & 4.256809 & 4.256810 \\
\hline & & & 1.0 & 0.703336 & 0.703347 & 1.685049 & 1.685046 & 4.358454 & 4.358455 \\
\hline & & & 10.0 & 0.850072 & 0.850076 & 1.921416 & 1.921416 & 4.751734 & 4.751734 \\
\hline & 1.0 & 0.6 & 0.1 & 0.461904 & 0.461893 & 1.165267 & 1.165266 & 2.480518 & 2.480518 \\
\hline & & & 1.0 & 0.700856 & 0.700855 & 1.197688 & 1.197686 & 2.634190 & 2.634190 \\
\hline & & & 10.0 & 0.801466 & 0.801468 & 1.238414 & 1.238412 & 2.891271 & 2.891271 \\
\hline \multirow[t]{9}{*}{0.6} & $\infty$ & 0.6 & 0.1 & 0.443601 & 0.443598 & 1.873050 & 1.873048 & 4.436802 & 4.436802 \\
\hline & & & 1.0 & 0.712292 & 0.712291 & 1.983124 & 1.983125 & 4.530416 & 4.530416 \\
\hline & & & 10.0 & 0.873798 & 0.873804 & 2.248240 & 2.248241 & 4.905603 & 4.905603 \\
\hline & & 1.0 & 0.1 & 0.423210 & 0.423220 & 1.621962 & 1.621961 & 4.295470 & 4.295470 \\
\hline & & & 1.0 & 0.671928 & 0.671929 & 1.732488 & 1.732486 & 4.395387 & 4.395387 \\
\hline & & & 10.0 & 0.809967 & 0.809974 & 1.984642 & 1.984642 & 4.784972 & 4.784972 \\
\hline & 1.0 & 0.6 & 0.1 & 0.439333 & 0.439333 & 1.162506 & 1.162500 & 2.520126 & 2.520126 \\
\hline & & & 1.0 & 0.671066 & 0.671065 & 1.184763 & 1.184762 & 2.684893 & 2.684893 \\
\hline & & & 10.0 & 0.773950 & 0.773954 & 1.212627 & 1.212624 & 2.960297 & 2.960296 \\
\hline
\end{tabular}

focus on the interface conditions between the two segments of the beam. For the displacement compatibility and force equilibrium conditions on the interface, one may go through Section 4.5 to handle the identical jump and scaled jump conditions with a modified jump command in the chebop system.

Several numerical examples with different classical boundary conditions and step locations are discussed. Consider a stepped beam consisting of two uniform segments $S_{1}$ and $S_{2}$. The length, width, thickness, mass per unit length, and cross-sectional moment of inertia are denoted by $l_{i}, b_{i}$, $h_{i}, m_{i}$, and $I_{i}$ of segment $S_{i}$, respectively. Both segments have the same Young's modulus $(E)$ and density $(\rho)$. Three configurations of stepped beam are presented and displayed in Figure 12:

Configuration $A: b_{2}=\varsigma b_{1}, h_{2}=h_{1}, m_{2} / m_{1}=\varsigma$ and $I_{2} / I_{1}$ $=1$

Configuration B: $b_{2}=b_{1}, h_{2}=\varsigma h_{1}, m_{2} / m_{1}=\varsigma$ and $I_{2} / I_{1}$ $=\varsigma^{3}$

Configuration C: $b_{2}=\varsigma b_{1}, h_{2}=\varsigma h_{1}, m_{2} / m_{1}=\varsigma^{2}$ and $I_{2} / I_{1}=\varsigma^{4}$

In this case, all the configurations of the stepped beam are compared to the results in [12]. Stepped beams with classical boundary conditions (clamped-free and simply supported) 


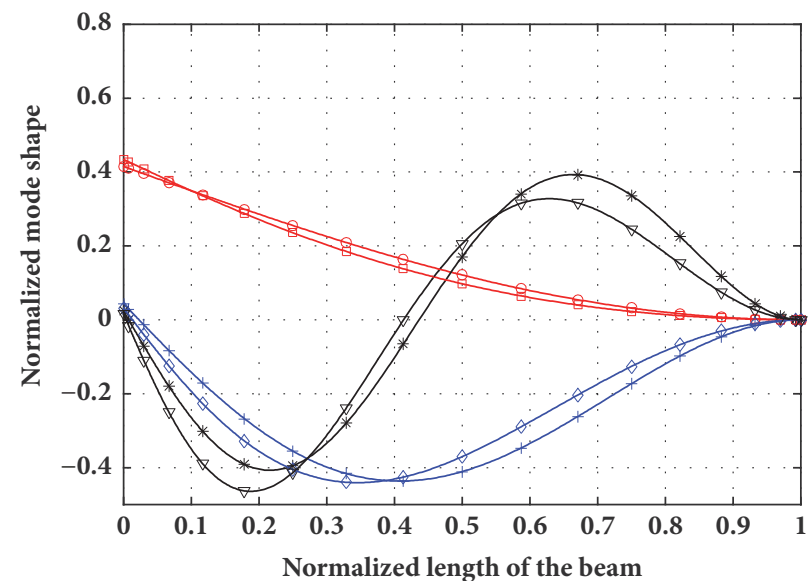

$\multimap$ 1st mode shape $(\alpha=1.2) \quad \square \quad$ 1st mode shape $(\alpha=2)$

+ 2nd mode shape $(\alpha=1.2) \multimap$ 2nd mode shape $(\alpha=2)$

$*$ 3rd mode shape $(\alpha=1.2) \rightarrow$ 3rd mode shape $(\alpha=2)$

FIGURE 9: The first three normalized mode shapes of a cantilever cone beam with different taper ratio of the beam $(\alpha)$.

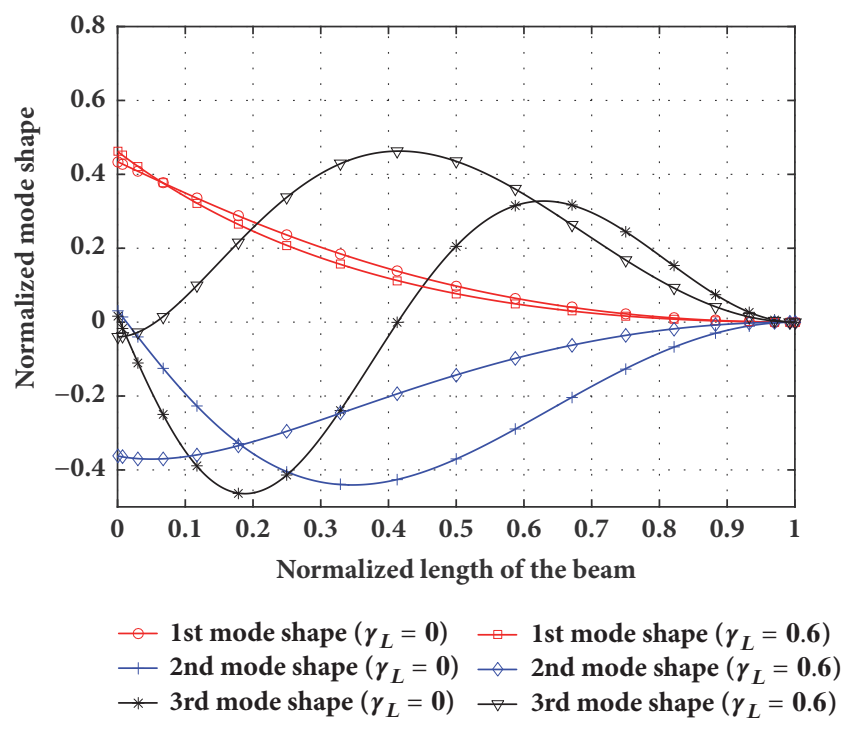

FIgURE 10: The first three normalized mode shapes of a cantilever cone beam with different rotatory inertias $\left(\gamma_{L}\right)$ at left end.

and various segment combinations are analyzed, with $R_{1}$ $\left(R_{1}=l_{1} /\left(l_{1}+l_{2}\right)\right)$ denoting the length ratio of the first segment. For $\varsigma=0.5$ and $R_{1}=0.25,0.375,0.625$, and 0.75 , the modal frequencies of each stepped beam are shown in Table 6. There are three types of stepped beams with two different boundary conditions and four different length ratio $\left(R_{1}\right)$, and the results of the first four natural frequencies are compared with those in [12]. We can see that the accuracy of the natural frequencies directly compete with [12] up to 4 decimal points. Figures 13-15 show the first three mode shapes of the stepped beams with $\varsigma=0.5$. In more detail, Figure 13 shows the mode shapes of clamped-free stepped beams $\mathrm{A}, \mathrm{B}$, and $\mathrm{C}$ with $R_{1}=0.375$, while Figure 14 shows the mode shapes of stepped beam A with $R_{1}=0.25$, as well as

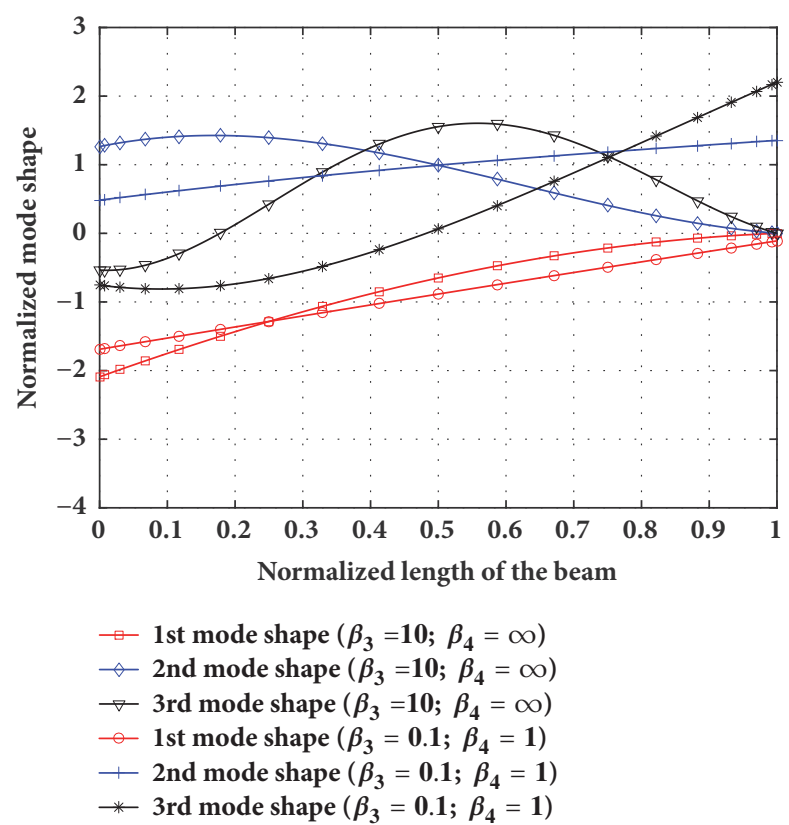

FIGURE 11: The first three normalized mode shapes of a cantilever cone beam with different clamped conditions $\left(\beta_{3}\right.$ and $\left.\beta_{4}\right)$ at right end.

the clamped-free and simply supported boundary conditions. Figure 15 shows the mode shapes of simply supported stepped beam A with $R_{1}=0.375$ and 0.75 . Consequently, for this twosegment EB beam example, the robustness and accuracy of the proposed approach can be verified by numerical results that directly compete with [12].

Next, as shown in Figure 16, we consider a simply supported uniform stepped EB beam with three segments; we illustrate a piezoelectric modal sensor as in [13]. The total length of the two-step beam is $l$, with the length and depth of each segment $l_{1}, l_{2}, l_{3}$ and $h_{1}, h_{2}, h_{3}$, respectively. Each segment has the same width, Young's modulus and density denoted by $b, E$, and $\rho$, respectively. The boundary condition of the stepped beam is simply supported and the scaling ratio from the left to the right of each segment is set at $\varsigma_{1}$ $=h_{2} / h_{1}=2$ and $\varsigma_{2}=h_{3} / h_{2}=0.5$. The position of the first step is located at $17 l / 38$ while the second is located at $21 l / 38$. The modal analysis of this two-step beam is carried out by our proposed approach. The first five natural frequencies are $3.2778,6.2920,9.8657,12.6356$, and 16.4596 , respectively, very close to the results in [13]. Moreover, Figure 17 shows the first five normalized mode shapes of this two-step beam.

Compared to the simply supported prismatic EB beam, doubling the thickness $\left(\varsigma_{1}=2\right)$ of the second segment will increase the nondimensional fundamental natural frequency from 3.14159 to 3.2778 (about 4\%). Also, this two-step beam example can be used to evaluate the influence of the natural frequency for applying adapter plate or fixture to the tunable resonant beam of a pyroshock simulator. Otherwise, the highorder mode shapes (17th-20th) plots of last two examples are provided in Supplementary Materials section (available here) for the interested reader. 
TABLE 6: The first four nondimensional natural frequencies for clamped-free and simply supported beams with a step ratio $\varsigma=0.5$.

\begin{tabular}{|c|c|c|c|c|c|c|c|}
\hline \multirow{2}{*}{ BCs } & \multirow{2}{*}{ Mode index } & \multirow{2}{*}{ Beam type } & \multirow{2}{*}{ Methods } & \multicolumn{4}{|c|}{ Length ratio of the first segment $\left(R_{1} / 1\right)$} \\
\hline & & & & 0.25 & 0.375 & 0.625 & 0.75 \\
\hline \multirow{24}{*}{ Clamped-free } & \multirow{6}{*}{1} & \multirow{2}{*}{ Beam-A } & Present & 2.0849 & 2.1526 & 2.1526 & 2.0849 \\
\hline & & & Ref. [9] & 2.0849 & 2.1526 & 2.1526 & 2.0849 \\
\hline & & \multirow{2}{*}{ Beam-B } & Present & 1.6687 & 1.8696 & 2.1193 & 2.0813 \\
\hline & & & Ref. [9] & 1.6687 & 1.8696 & 2.1193 & 2.0813 \\
\hline & & \multirow{2}{*}{ Beam-C } & Present & 1.7151 & 1.9777 & 2.3651 & 2.2454 \\
\hline & & & Ref. [9] & 1.7151 & 1.9777 & 2.3651 & 2.2454 \\
\hline & \multirow{6}{*}{2} & \multirow{2}{*}{ Beam-A } & Present & 4.9153 & 4.7950 & 4.7950 & 4.9153 \\
\hline & & & Ref. [9] & 4.9153 & 4.7950 & 4.7950 & 4.9153 \\
\hline & & \multirow{2}{*}{ Beam-B } & Present & 3.9528 & 3.9387 & 4.0976 & 4.6760 \\
\hline & & & Ref. [9] & 3.9528 & 3.9387 & 4.0976 & 4.6760 \\
\hline & & \multirow{2}{*}{ Beam-C } & Present & 4.1395 & 4.1638 & 3.9885 & 4.8251 \\
\hline & & & Ref. [9] & 4.1395 & 4.1638 & 3.9885 & 4.8521 \\
\hline & \multirow{6}{*}{3} & \multirow{2}{*}{ Beam-A } & Present & 7.9045 & 7.8343 & 7.8343 & 7.9045 \\
\hline & & & Ref. [9] & 7.9045 & 7.8343 & 7.8343 & 7.9045 \\
\hline & & \multirow{2}{*}{ Beam-B } & Present & 6.1635 & 6.1399 & 6.8479 & 6.9380 \\
\hline & & & Ref. [9] & 6.1635 & 6.1399 & 6.8479 & 6.9380 \\
\hline & & \multirow{2}{*}{ Beam-C } & Present & 6.3841 & 6.0002 & 7.0045 & 6.7812 \\
\hline & & & Ref. [9] & 6.8341 & 6.0002 & 7.0045 & 6.7812 \\
\hline & \multirow{6}{*}{4} & \multirow{2}{*}{ Beam-A } & Present & 10.9711 & 11.0414 & 11.0414 & 10.9711 \\
\hline & & & Ref. [9] & 10.9711 & 11.0414 & 11.0414 & 10.9711 \\
\hline & & Beam-B & Present & 8.3054 & 8.8732 & 9.4841 & 9.9945 \\
\hline & & Dedin-D & Ref. [9] & 8.3054 & 8.8733 & 9.4841 & 9.9945 \\
\hline & & Beam-C & Present & 8.2156 & 8.8775 & 9.3342 & 10.1310 \\
\hline & & 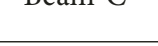 & Ref. [9] & 8.2156 & 8.8775 & 9.3342 & 10.1310 \\
\hline & & Beam-A & Present & 3.1160 & 3.0890 & 3.0890 & 3.1160 \\
\hline & & 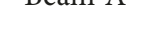 & Ref. [9] & 3.1160 & 3.0890 & 3.0890 & 3.1160 \\
\hline & 1 & Beam-B & Present & 2.2270 & 2.2637 & 2.5443 & 2.8238 \\
\hline & & & Ref. [9] & 2.2270 & 2.2637 & 2.5443 & 2.8238 \\
\hline & & Beam-C & Present & 2.1602 & 2.1257 & 2.3111 & 2.6160 \\
\hline & & Dedinter ty & Ref. [9] & 2.1602 & 2.1257 & 2.3111 & 2.6160 \\
\hline & & Beam-A & Present & 6.2218 & 6.2831 & 6.2831 & 6.2218 \\
\hline & & Dedint & Ref. [9] & 6.2218 & 6.2831 & 6.2831 & 6.2218 \\
\hline & 2 & Beam-B & Present & 4.5706 & 4.9160 & 5.5403 & 5.4805 \\
\hline & & & Ref. [9] & 4.5706 & 4.9160 & 5.5403 & 5.4805 \\
\hline & & Beam-C & Present & 4.3995 & 4.7788 & 5.7147 & 5.4039 \\
\hline Simply supported & & & Ref. [9] & 4.3995 & 4.7788 & 5.7147 & 5.4039 \\
\hline 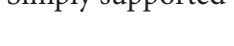 & & Beam-A & Present & 9.4248 & 9.4667 & 9.4667 & 9.4248 \\
\hline & & Dentint & Ref. [9] & 9.4248 & 9.4667 & 9.4667 & 9.4248 \\
\hline & 3 & Beam-B & Present & 7.0984 & 7.6854 & 8.1049 & 8.6897 \\
\hline & J & 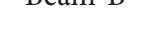 & Ref. [9] & 7.0984 & 7.6854 & 8.1049 & 8.6897 \\
\hline & & Beam-C & Present & 6.9661 & 7.7817 & 7.9441 & 8.8739 \\
\hline & & & Ref. [9] & 6.9661 & 7.7817 & 7.9441 & 8.8739 \\
\hline & & Beam-A & Present & 12.6251 & 12.5075 & 12.5075 & 12.6251 \\
\hline & & Deantin & Ref. [9] & 12.6253 & 12.5076 & 12.5076 & 12.6253 \\
\hline & 4 & Beam-B & Present & 9.6906 & 9.9389 & 10.8726 & 11.4763 \\
\hline & $T$ & 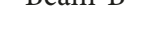 & Ref. [9] & 9.6908 & 9.9390 & 10.8727 & 11.4765 \\
\hline & & Beam-C & Present & 9.6683 & 10.0408 & 10.9903 & 11.4005 \\
\hline & & Detant & Ref. [9] & 9.6684 & 10.0409 & 10.9905 & 11.4007 \\
\hline
\end{tabular}



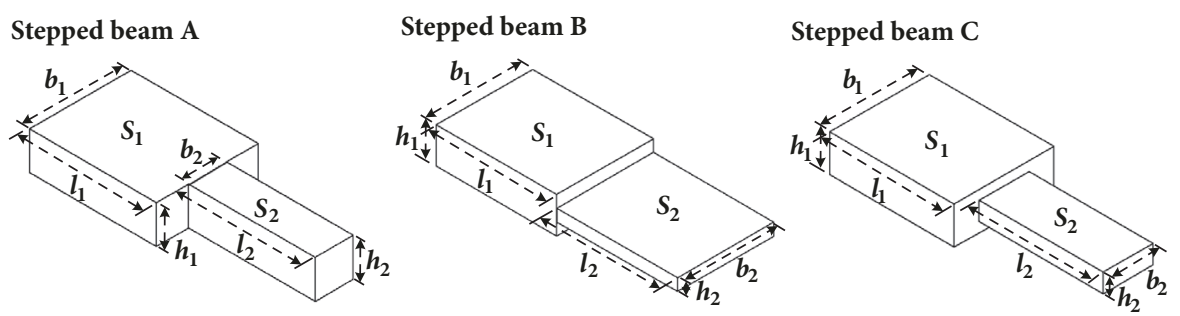

FIGURE 12: Three cases for a stepped beam.

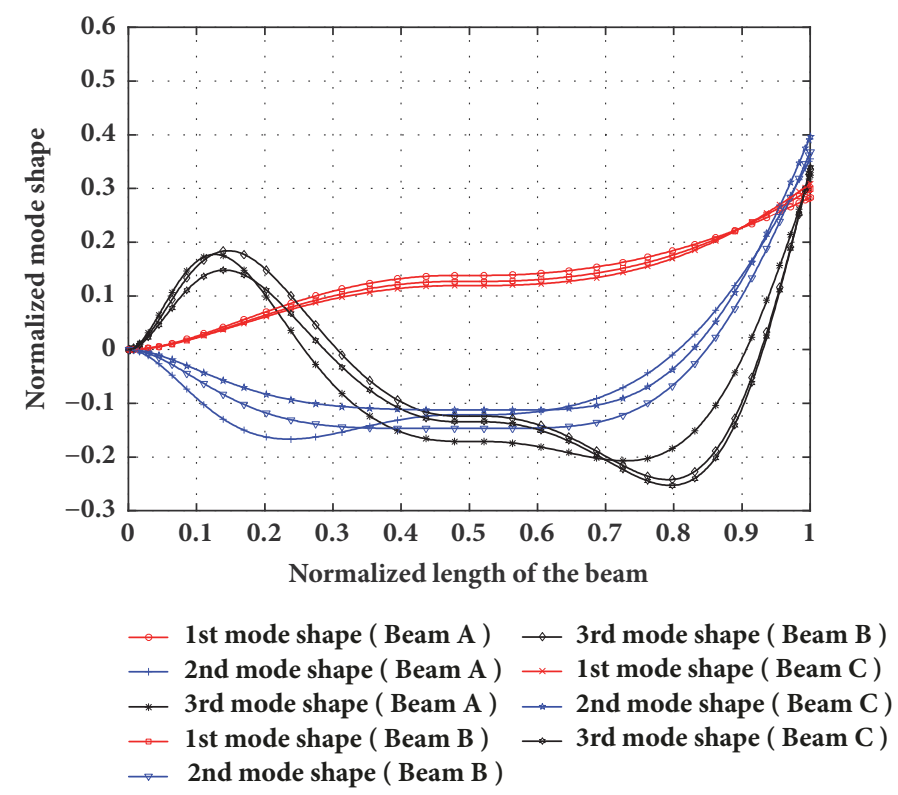

FIGURE 13: The first three normalized mode shapes of clamped-free stepped beams A, B, and C ( $\varsigma=0.5$ and $\left.R_{1}=0.375\right)$.

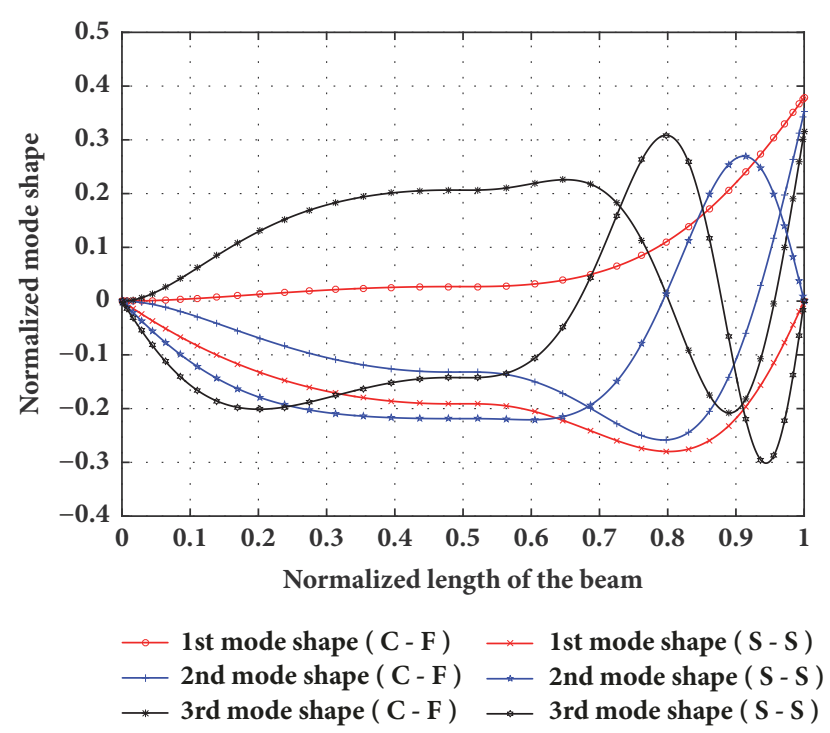

FIGURE 14: The first three normalized mode shapes of stepped beam A with clamped-free and simply supported boundary conditions ( $\varsigma$ $=0.5$ and $R_{1}=0.25$ ).

\section{Conclusion and Discussion}

The Chebyshev spectral method with the null space approach for the modal solutions of a nonprismatic EB beam obeying general boundary and interface conditions has been presented. A unique and more general asymptotic modal solution for the higher-order modes of partially clamped EB beam has also been derived. With the chebop system in the Chebfun toolbox, the symbolic formulation for the governing equation and the boundary as well as interface conditions of an EB beam has been converted to a differential system matrix preconditioned with the null space approach. Results at different boundary or interface conditions for nonprismatic EB beams are benchmarked with those reported in the literature using alternative methods and validate the general modal solutions for EB beams with eigenvalue embedded boundary conditions, higher-order modes, and nonprismatic cross-sections. For further applications, it is recommended that tunable resonant beams for simulating pyroshock should be evaluated by the proposed approach, thereby minimizing the required trials and errors for tuning the shock spectrum knee frequency of the resonant beam. 


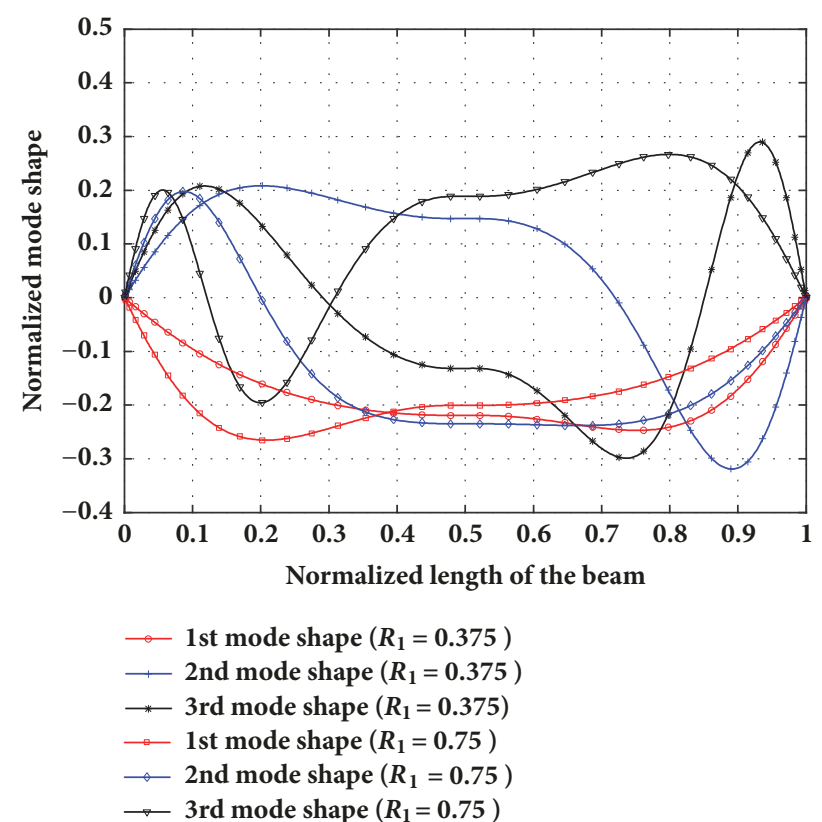

FIGURE 15: The first three normalized mode shapes of simply supported stepped beam A $\left(R_{1}=0.375,0.75\right.$, and $\left.\varsigma=0.5\right)$.

\section{Appendix}

\section{Higher-Order Asymptotic Modal}

\section{Solutions for an EB Beam with Partially Clamped Boundary Conditions}

Substituting the characteristic function in (22) into the boundary conditions in (21), with $\Lambda=k(\omega) l$, yields the following homogeneous matrix equation (A.1) in terms of $C_{1}$ to $\mathrm{C}_{4}$,

$$
\left[\begin{array}{cccc}
\beta_{1} & \Lambda_{i} & \beta_{1} & -\Lambda_{i} \\
\Lambda_{i}^{3} & -\beta_{2} & -\Lambda_{i}^{3} & -\beta_{2} \\
\beta_{3} C-\Lambda_{i} S & -\beta_{3} S-\Lambda_{i} C & \beta_{3} C_{H}+\Lambda_{i} S_{H} & \Lambda_{i} C_{H}+\beta_{3} S_{H} \\
\Lambda_{i}^{3} C+\beta_{4} S & \beta_{4} C-\Lambda_{i}^{3} S & S_{H}-\Lambda_{i}^{3} C_{H} & \beta_{4} C_{H}-\Lambda_{i}^{3} S_{H}
\end{array}\right]\left\{\begin{array}{l}
C_{1} \\
C_{2} \\
C_{3} \\
C_{4}
\end{array}\right\}=0
$$

where $S, C, S_{H}$, and $C_{H}$ represent $\sin \left(\Lambda_{i}\right), \cos \left(\Lambda_{i}\right), \sinh \left(\Lambda_{i}\right)$, and $\cosh \left(\Lambda_{i}\right)$, respectively.

The determinant of the LHS coefficient matrix is set to zero to obtain the characteristic equation, from which the nondimensional natural frequencies $\Lambda_{i}=k_{i} l$ can be determined from (A.2) as follows:

$$
\begin{aligned}
& P_{0} \beta_{1} \beta_{2} \beta_{3} \beta_{4}+P_{1} \beta_{2} \beta_{4}\left(\beta_{1}+\beta_{3}\right) \Lambda_{i}+P_{2} \beta_{2} \beta_{4} \Lambda_{i}^{2} \\
& +P_{3} \beta_{1} \beta_{3}\left(\beta_{2}+\beta_{4}\right) \Lambda_{i}^{3} \\
& +\left[P_{4}\left(\beta_{1} \beta_{2}+\beta_{3} \beta_{4}\right)+\left(P_{4}-P_{0}\right)\left(\beta_{1} \beta_{4}+\beta_{2} \beta_{3}\right)\right] \Lambda_{i}^{4} \\
& \quad-P_{1}\left(\beta_{2}+\beta_{4}\right) \Lambda_{i}^{5}-P_{2} \beta_{1} \beta_{3} \Lambda_{i}^{6}-P_{3}\left(\beta_{1}+\beta_{3}\right) \Lambda_{i}^{7} \\
& +P_{0} \Lambda_{i}^{8}=0
\end{aligned}
$$

where

$$
\begin{aligned}
& P_{0}=1-C C_{H} ; \\
& P_{1}=S C_{H}-C S_{H} ; \\
& P_{2}=2 S S_{H} ; \\
& P_{3}=C S_{H}+S C_{H} ; \\
& P_{4}=1+C C_{H} .
\end{aligned}
$$

Then, one may obtain the solutions of $C_{1}$ to $C_{4}$ with (A.1) as

$$
\begin{aligned}
C_{1}= & \left(C-C_{H}\right) \Lambda_{i}^{5}+\beta_{3}\left(S-S_{H}\right) \Lambda_{i}^{4}+2 \beta_{2} S_{H} \Lambda_{i}^{2} \\
& +\left[\beta_{1} \beta_{2}\left(C_{H}+C\right)+2 \beta_{2} \beta_{3} C_{H}\right] \Lambda_{i} \\
& +\beta_{1} \beta_{2} \beta_{3}\left(S+S_{H}\right), \\
C_{2}= & \left(S_{H}-S\right) \Lambda_{i}^{5}+\left[\beta_{3}\left(C_{H}+C\right)+2 \beta_{1} C_{H}\right] \Lambda_{i}^{4} \\
& +2 \beta_{1} \beta_{3} S_{h} \Lambda_{i}^{3} \\
& -\left[\beta_{1} \beta_{2}\left(S_{H}+S\right)+2 \beta_{2} \beta_{3} C_{H}\right] \Lambda_{i} \\
& +\beta_{1} \beta_{2} \beta_{3}\left(C-C_{H}\right), \\
C_{3}= & \left(C-C_{H}\right) \Lambda_{i}^{5}+\beta_{3}\left(S-S_{H}\right) \Lambda_{i}^{4}+2 \beta_{2} S \Lambda_{i}^{2} \\
& -\left[\beta_{1} \beta_{2}\left(C_{H}+C\right)+2 \beta_{2} \beta_{3} C\right] \Lambda_{i} \\
& -\beta_{1} \beta_{2} \beta_{3}\left(S+S_{H}\right), \\
C_{4}= & \left(S_{H}-S\right) \Lambda_{i}^{5}+\left[\beta_{3}\left(C_{H}+C\right)+2 \beta_{1} C\right] \Lambda_{i}^{4} \\
& +2 \beta_{1} \beta_{3} S \Lambda_{i}^{2}+\beta_{1} \beta_{2}\left(S_{H}+S\right) \Lambda_{i} \\
& +\beta_{1} \beta_{2} \beta_{3}\left(C_{H}-C\right) .
\end{aligned}
$$

Introducing $C_{1}$ to $C_{4}$ into (21), we obtain the following analytical solution of the $i$-th mode shape for partially clamped EB beam:

$$
\begin{aligned}
\psi_{i, \text { exact }}(x)=\sin \left(k_{i} x\right)\left[\left(C-C_{H}\right) \Lambda_{i}^{5}\right. \\
+\left(S-S_{H}\right) \beta_{3} \Lambda_{i}^{4}+2 \beta_{2} S_{H} \Lambda_{i}^{2} \\
+\left(\beta_{1} C_{H}+\beta_{1} C+2 \beta_{3} C_{H}\right) \beta_{2} \Lambda_{i} \\
\left.+\left(S_{H}+S\right) \beta_{1} \beta_{2} \beta_{3}\right]+\cos \left(k_{i} x\right)\left[\left(S_{H}-S\right) \Lambda_{i}^{5}\right. \\
+\left(2 \beta_{1} C_{H}+\beta_{3} C_{H}+\beta_{3} C\right) \Lambda_{i}^{4}+2 \beta_{2} \beta_{3} S_{H} \Lambda_{i}^{3} \\
\left.+\left(S_{h}+S\right) \beta_{1} \beta_{2} \Lambda_{i}-\left(C_{H}+C\right) \beta_{1} \beta_{2} \beta_{3}\right] \\
+\sinh \left(k_{i} x\right)\left[\left(C-C_{H}\right) \Lambda_{i}^{5}+\left(S-S_{H}\right) \beta_{3} \Lambda_{i}^{4}\right. \\
+2 \beta_{2} S \Lambda_{i}^{2}-\left(\beta_{1} C+\beta_{1} C_{H}+2 \beta_{3} C\right) \beta_{2} \Lambda_{i} \\
\left.+\left(S+S_{H}\right) \beta_{1} \beta_{2} \beta_{3}\right]+\cosh \left(k_{i} x\right)\left[\left(S_{H}-S\right) \Lambda_{i}^{5}\right. \\
+\left(2 \beta_{1} \mathrm{C}+\beta_{3} C_{H}+\beta_{3} C\right) \Lambda_{i}^{4}+2 \beta_{1} \beta_{3} S \Lambda_{i}^{3} \\
\left.+\left(S_{H}+S\right) \beta_{1} \beta_{2} \Lambda_{i}+\left(C_{H}-C\right) \beta_{1} \beta_{2} \beta_{3}\right]
\end{aligned}
$$




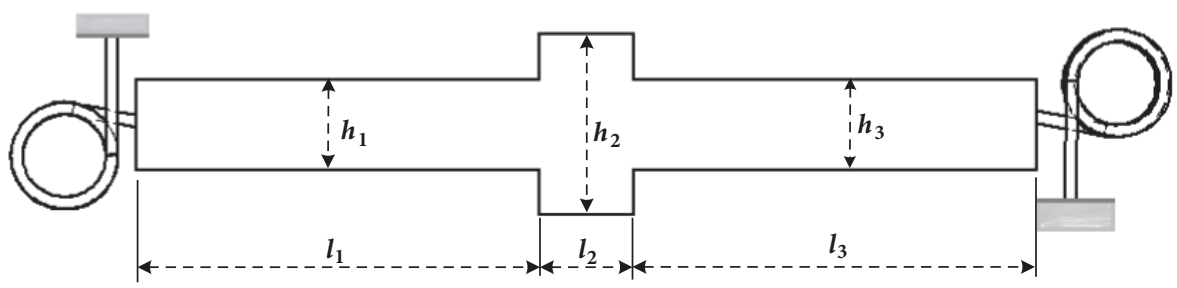

FIGURE 16: Uniform three stepped EB beams with S-S BCs.

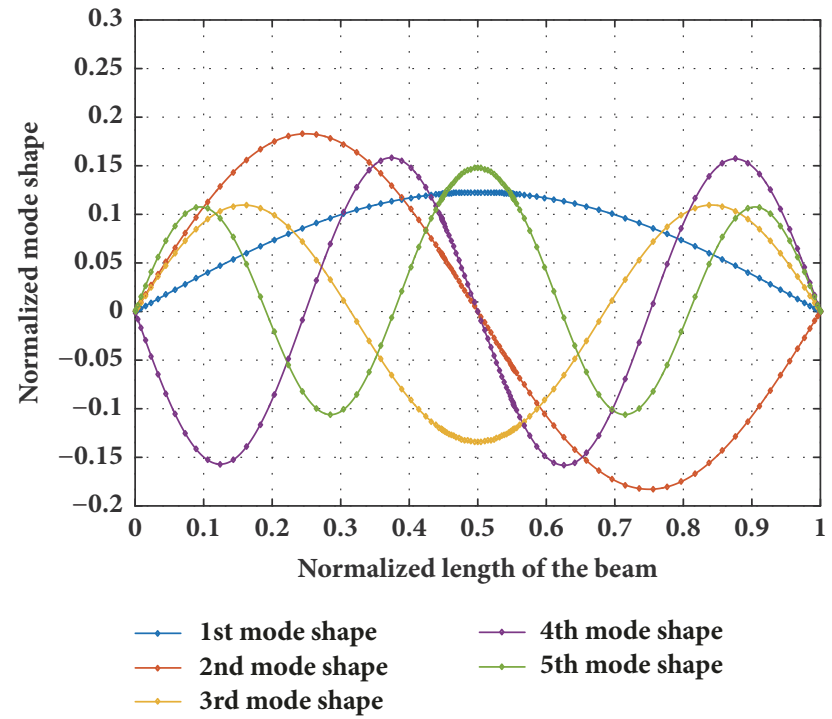

FIGURE 17: The first five normalized mode shapes for the beam shown in Figure 16.

For the first 12 modes, using (A.2) and (A.3), a numerical root-finder may search the nondimensional natural frequency $\Lambda_{i}$ for the corresponding mode and obtain the mode shape by using (A.4) and (A.5). However, (A.2) and (A.5) permit the numerical evaluation for the first 12 modes (e.g., $\left.\Lambda_{i} \leq 12 \pi\right)$ or so; otherwise both equations are numerically ill-conditioned due to the double precision floating point arithmetic of the MATLAB environment. For $\Lambda_{i} \geq 13 \pi$, an asymptotic simplification approach is used as follows:

$$
C_{H}-\exp \left(-\Lambda_{i}\right)=S_{H} \gg C \text { or } S, \text { for } \Lambda_{i} \geq 13 \pi
$$

Substituting (A.6) into (A.2) and (A.5), with the aid of symbolic computation software, we obtain the asymptotic higher-order modal solutions for the partially clamped EB beam as

$$
\begin{aligned}
& \psi_{i, \text { asymp }}(x)=\sin \left(k_{i} x\right)\left[-\Lambda_{i}^{5}-\beta_{3} \Lambda_{i}^{4}+2 \beta_{2} \Lambda_{i}^{2}\right. \\
& \left.+\left(\beta_{1} \beta_{2}+2 \beta_{3} \beta_{2}\right) \Lambda_{i}+\beta_{3} \beta_{2} \beta_{1}\right]+\cos \left(k_{i} x\right)\left[\Lambda_{i}^{5}\right. \\
& \left.+\left(\beta_{3}+2 \beta_{1}\right) \Lambda_{i}^{4}+2 \beta_{1} \beta_{3} \Lambda_{i}^{3}-\beta_{1} \beta_{2} \Lambda_{i}-\beta_{3} \beta_{2} \beta_{1}\right] \\
& +\mathrm{e}^{-k_{i} x}\left(\beta_{3} \beta_{2} \beta_{1}+\Lambda_{i}^{5}+\beta_{3} \Lambda_{i}^{4}+\beta_{1} \beta_{2} \Lambda_{i}\right)
\end{aligned}
$$

$$
\begin{aligned}
& +\mathrm{e}^{-k_{i}(l-x)}\left\{(C-S) \Lambda_{i}^{5}+\left[\beta_{3}(S+C)+2 \beta_{1} C\right] \Lambda_{i}^{4}\right. \\
& +2 \beta_{1} \beta_{3} S \Lambda_{i}^{3}+\beta_{2} S \Lambda_{i}^{2} \\
& \left.+\beta_{2}\left[\beta_{1}(S-C)-2 \beta_{3} C\right] \Lambda_{i}-\beta_{1} \beta_{2} \beta_{3}(S+C)\right\},
\end{aligned}
$$

which reduces (A.3) to the following $P_{0}$ to $P_{4}$ asymptotic coefficients in the characteristic equation (A.2):

$$
\begin{aligned}
& P_{0}=-C ; \\
& P_{1}=S-C ; \\
& P_{2}=2 S ; \\
& P_{3}=C+S ; \\
& P_{4}=C .
\end{aligned}
$$

With (A.2) and (A.8), one may use a numerical root-finder in searching the nondimensional natural frequency $\Lambda_{i}$ for the corresponding higher-order mode and obtain the mode shape using (A.7).

It should be noted that, in Table 2 of [8], there are ten formulas for the natural frequencies and mode shapes for ten corresponding classical boundary conditions. However, by using (A.2) and (A.8) as well as feasible values for $\beta_{i}$, this appendix provides a unique set of generalized modal solution. For the case of a simply supported beam $\left(\beta_{1}=\beta_{3}\right.$ $=0$ and $\beta_{2}=\beta_{4}=\infty$ ), with (A.2) and (A.8), the higher-order nondimensional natural frequency is $\Lambda_{i}=n \pi\left(P_{2}=0\right)$, and the corresponding mode shape is $\sin \left(k_{i} x\right)$ by (A.7).

\section{Data Availability}

The data used to support the findings of this study are available from the corresponding author upon request.

\section{Conflicts of Interest}

The authors declare that they have no conflicts of interest.

\section{Acknowledgments}

The authors would like to thank Professor Michalopoulos for providing helpful suggestions that improved the presentation and content of this paper. 


\section{Supplementary Materials}

File name: Supp_Mat.zip. S1: uniformeuler_review.m. Matlab M-file demonstrates eigensolution of an EB beam under generalized boundary conditions by using proposed Chebyshev spectral method and null space approach. S2: FIG 8_S-17_S.pdf. FIG 8_S-FIG_11S are plots of the higher-order mode shapes (17th-20th) in numerical example of Section 5.3. FIG 13_S-FIG_15S and FIG_17S are plots of the higher-order mode shapes (17th-20th) in numerical example of Section 5.4. (Supplementary Materials)

\section{References}

[1] Ping Lou and Qing-yuan Zeng, "Formulation of Equations of Motion for a Simply Supported Bridge under a Moving Railway Freight Vehicle," Shock and Vibration, vol. 14, pp. 1-18, 2007.

[2] Ki-Young Koo and Jin-Hak Yi, "Substructural Identification of Flexural Rigidity for Beam-Like Structures," Shock and Vibration, vol. 2015, pp. 1-15, 2015.

[3] Q. Mao, "Free vibration analysis of elastically connected multiple-beams by using the Adomian modified decomposition method," Journal of Sound and Vibration, vol. 331, no. 11, pp. 2532-2542, 2012.

[4] P. F. Pai, L. Huang, S. H. Gopalakrishnamurthy, and J. H. Chung, "Identification and applications of boundary effects in beams," International Journal of Solids and Structures, vol. 41, no. 11-12, pp. 3053-3080, 2004.

[5] N. Olgac and N. Jalili, "Modal analysis of flexible beams with delayed resonator vibration absorber: Theory and experiments," Journal of Sound and Vibration, vol. 218, no. 2, pp. 307-331, 1998.

[6] E. B. Magrab, Vibrations of elastic systems: with applications to MEMS and NEMS, vol. 184, Springer Science and Business Media, 2012.

[7] J. W. Jeong, J. H. Lim, K. W. Kim, and J. J. Lee, "Development of a Point Pyroshock Source Simulator," Shock and Vibration, vol. 2017, pp. 1-16, 2017.

[8] P. J. P. Gonçalves, M. J. Brennan, and S. J. Elliott, "Numerical evaluation of high-order modes of vibration in uniform EulerBernoulli beams," Journal of Sound and Vibration, vol. 301, no. 3-5, pp. 1035-1039, 2007.

[9] K. C. Cha, N. Wang, and J. Y. Liao, "Dynamics and cutting stability of the dynamically loaded worktable subjected to simply supported conditions," The International Journal of Advanced Manufacturing Technology, vol. 71, no. 1-4, pp. 605-620, 2014.

[10] J.-C. Hsu, H.-Y. Lai, and C.-K. Chen, "An innovative eigenvalue problem solver for free vibration of uniform Timoshenko beams by using the Adomian modified decomposition method," Journal of Sound and Vibration, vol. 325, no. 1-2, pp. 451-470, 2009.

[11] H.-Y. Lai, C. K. Chen, and J.-C. Hsu, "Free vibration of nonuniform Euler-Bernoulli beams by the Adomian modified decomposition method," Computer Modeling in Engineering \& Sciences, vol. 34, no. 1, pp. 87-113, 2008.

[12] Q. Mao and S. Pietrzko, "Free vibration analysis of stepped beams by using Adomian decomposition method," Applied Mathematics and Computation, vol. 217, no. 7, pp. 3429-3441, 2010.

[13] Q. Mao, "Design of piezoelectric mode sensor for non-uniform Euler-Bernoulli beams with rectangular cross-section by using differential transformation method," Mechanical Systems and Signal Processing, vol. 33, pp. 142-154, 2012.

[14] M. Attar, "A transfer matrix method for free vibration analysis and crack identification of stepped beams with multiple edge cracks and different boundary conditions," International Journal of Mechanical Sciences, vol. 57, no. 1, pp. 19-33, 2012.

[15] K. Sarkar and R. Ganguli, "Modal tailoring and closed-form solutions for rotating non-uniform Euler-Bernoulli beams," International Journal of Mechanical Sciences, vol. 88, pp. 208220, 2014.

[16] J. Shen, T. Tang, and L. Wang, "Applications in Multi-Dimensional Domains," in Spectral Methods, vol. 41 of Springer Series in Computational Mathematics, pp. 367-413, Springer Berlin Heidelberg, Berlin, Heidelberg, 2011.

[17] L. Fox and I. B. Parker, Chebyshev Polynomials in Numerical Analyis, Oxford University Press, 1st edition, 1968.

[18] J. Lee and W. W. Schultz, "Eigenvalue analysis of Timoshenko beams and axisymmetric Mindlin plates by the pseudospectral method," Journal of Sound and Vibration, vol. 269, no. 3-5, pp. 609-621, 2004.

[19] M. S. Sari and E. A. Butcher, "Free vibration analysis of nonrotating and rotating Timoshenko beams with damaged boundaries using the Chebyshev collocation method," International Journal of Mechanical Sciences, vol. 60, no. 1, pp. 1-11, 2012.

[20] N. Wattanasakulpong and Q. Mao, "Dynamic response of Timoshenko functionally graded beams with classical and nonclassical boundary conditions using Chebyshev collocation method," Composite Structures, vol. 119, pp. 346-354, 2014.

[21] W. Y. Wang, J. Y. Liao, and L. W. Hourng, "Matrix-based Chebyshev spectral approach to dynamic analysis of non-uniform timoshenko beams," Structural Engineering and Mechanics, vol. 39, no. 5, pp. 669-682, 2011.

[22] L. N. Trefethen, Spectra Methods in MATLAB, SIAM, Philadelphia, Pennsylvania, 1st edition, 2001.

[23] T. A. Driscoll, "Automatic spectral collocation for integral, integro-differential, and integrally reformulated differential equations," Journal of Computational Physics, vol. 229, no. 17, pp. 5980-5998, 2010.

[24] "The Chebfun Team, University of Oxford," http://www .chebfun.org/.

[25] T. A. Driscoll, F. Bornemann, and L. N. Trefethen, "The chebop system for automatic solution of differential equations," BIT Numerical Mathematics, vol. 48, no. 4, pp. 701-723, 2008.

[26] T. A. Driscoll and N. Hale, "Rectangular spectral collocation," IMA Journal of Numerical Analysis (IMAJNA), vol. 36, no. 1, pp. 108-132, 2016.

[27] J. L. Aurentz and L. N. Trefethen, "Block operators and spectral discretizations," SIAM Review, vol. 59, no. 2, pp. 423-446, 2017.

[28] B. Smith, R. Laoulache, and A. Heryudono, "Implementation of Neumann boundary condition with influence matrix method for viscous annular flow using pseudospectral collocation," Journal of Computational and Applied Mathematics, vol. 285, pp. 100-115, 2015.

[29] J. H. Wilkinson, The Algebraic Problem, Oxford University Press, Oxford, UK, 1965.

[30] Y. Saad, Numerical Methods for Large Eigenvalue Problems, Classics in Applied Mathematics, Society for Industrial and Applied Mathematics (SIAM), Philadelphia, PA, USA, 2nd edition, 2011.

[31] P.-y. Nie, "A null space method for solving system of equations," Applied Mathematics and Computation, vol. 149, no. 1, pp. 215226, 2004. 
[32] C. L. Mitsas, "Data reconciliation and variable classification by null space methods," Measurement, vol. 43, no. 5, pp. 702-707, 2010.

[33] C. Felippa and K. Park, "The construction of free-free flexibility matrices for multilevel structural analysis," Computer Methods Applied Mechanics and Engineering, vol. 191, no. 19-20, pp. 21392168, 2002. 


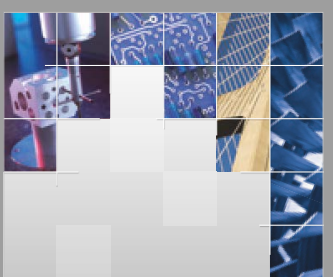

\section{Enfincering}
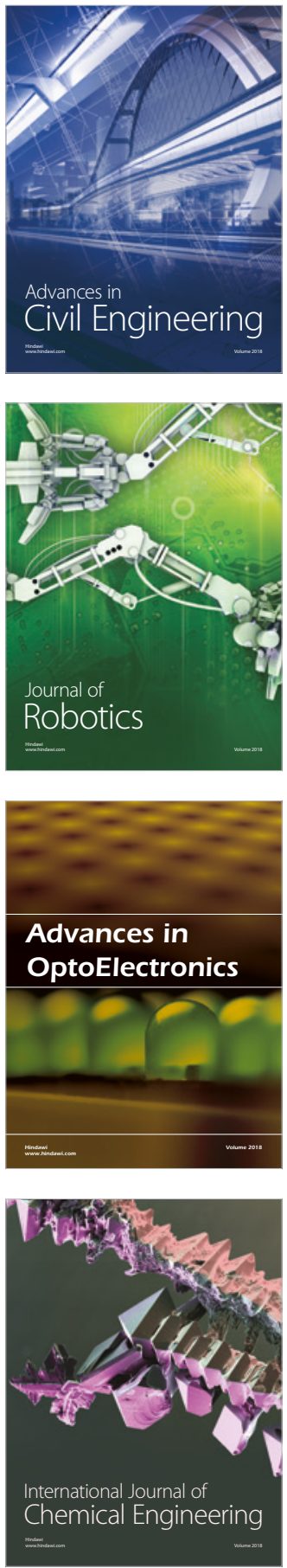

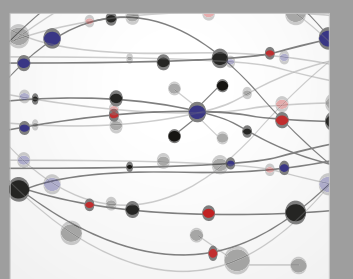

\section{Rotating \\ Machinery}

The Scientific World Journal

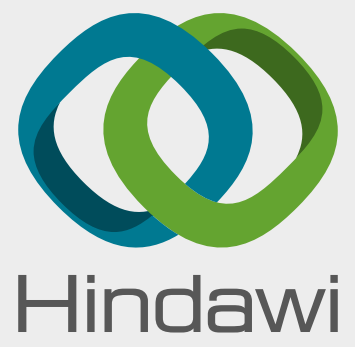

Submit your manuscripts at

www.hindawi.com
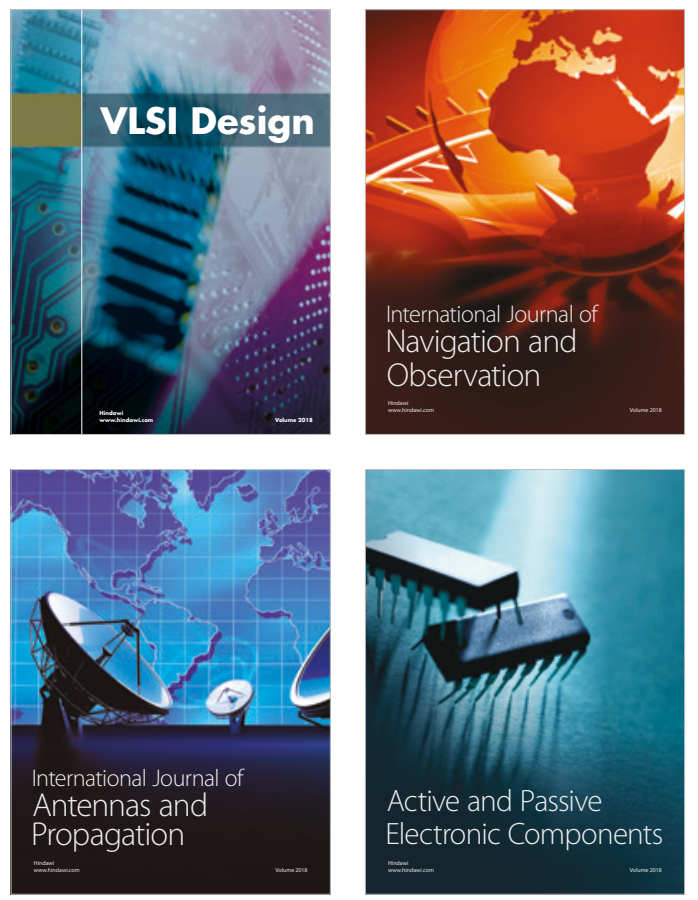
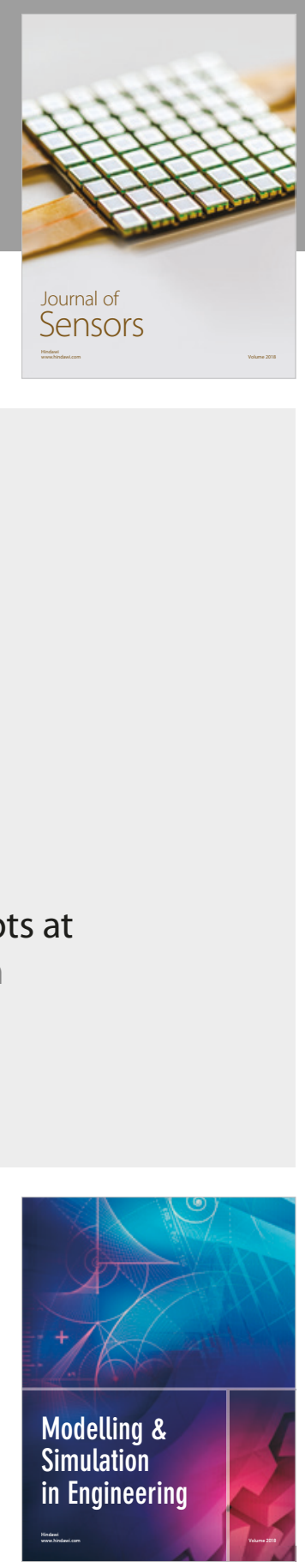

\section{Advances \\ Multimedia}
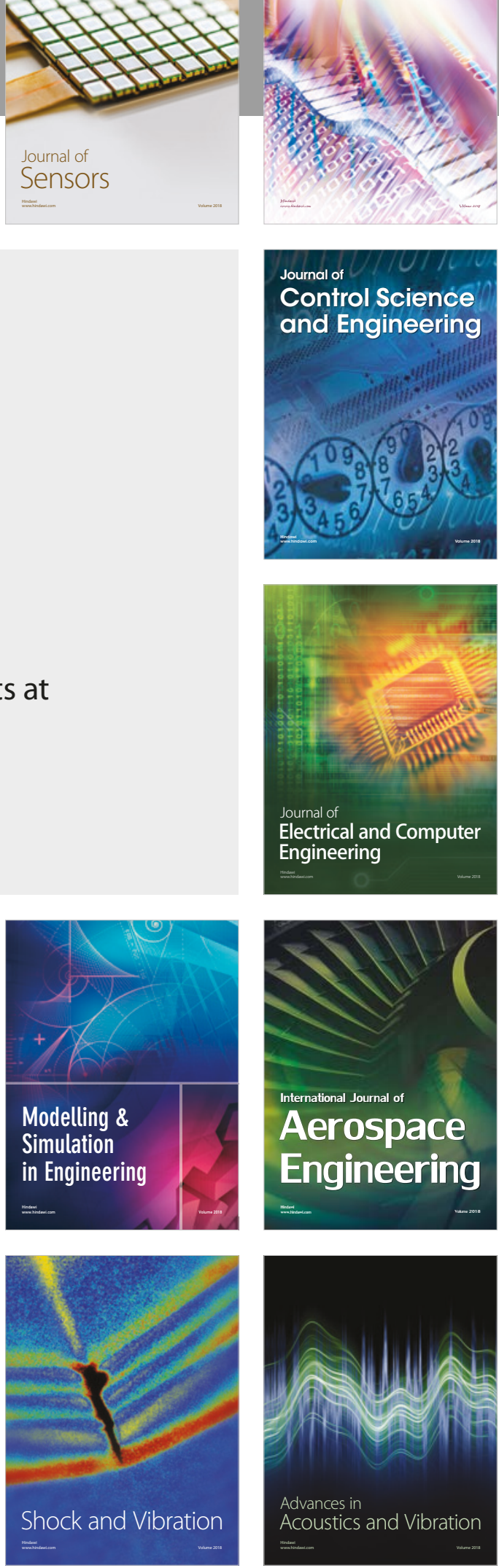